\title{
Die Cylinderfunctionen erster und zweiter Art.
}

Von Hermanx Hankel in Erlangen.

Die bekannte Differentialgleichung

$$
\frac{\partial^{2} R}{\partial x^{2}}+\frac{1}{x} \frac{\partial R}{\partial x}+\left(1-\frac{n^{2}}{x^{2}}\right) R=0,
$$

welche in fast allen mathematisch-physikalischen Untersuchungen auftritt, die sich anf einen gegen eine Axe symmetrischen Zustand beziehen, wird im Allgemeinen, fuir ein beliebiges complexes $x$ und $n$, durch zwei Functionen

$$
J^{n}(x) \text { and } J^{-n}(x)
$$

integrirt, wenn diese Cylinderfunctionen*) erster Art durch

$$
J^{n}(x)=\left(\frac{x}{2}\right)^{n} \sum_{x=0}^{\infty} \frac{(-1)^{p}}{\Gamma(n+p+1) \Gamma(p+1)}\left(\frac{x}{2}\right)^{2 p}
$$

definirt werden. Nur in dem Falle, dass $n$ eine positive ganze Zahl ist, fallen diese beiden sonst verschiedenen Lösungen zusammen und es entsteht somit die Aufgabe, neben $J^{n}(x)$ noch eine andere particuläre Lösung der Differentialgleichung aufzusuchen, die ich in Uebereinstimmung mit Hrn. C. Neumann**), der kürzlich diese Cylinderfunctionen $\mathrm{zweiter}$ Art einer ausführlichen Betrachtung unterzogen hat, mit $Y^{n}(x)$ bezeichne. Letzterer hat $Y^{n}(x)$ in zwei Theile zerlegt: $Y^{n}(x)=L^{n}(x)+E^{n}(x)$, wo:

$$
L^{n}(x)=J^{n}(x) \cdot \log x-\frac{\Gamma(n+1)}{2} \sum_{p=0}^{n-1} \frac{2^{n-p}}{n-p} \frac{1}{\Gamma(p+1)} \frac{J^{p}(x)}{x^{n-p}}
$$

gesetzt wird und

$E^{n}(x)=-\left(1+\frac{1}{2}+. .+\frac{1}{n}\right) J^{n}(x)-4 \sum_{p=1}^{\infty}(-1)^{n} \frac{n+2 p}{2 n+2 p} \frac{J^{n+2 p}(x)}{2^{2 p}}$

Diesen letzten Theil hat Hr. Lommel ${ }^{*}$ ) in die Form:

*) Ich schliesse mich hier in der Bezeichnung dem passenden Vorschlage Hrn. Heine's an (Crelle Journ. t.

**) Theorie der Bessel'schen Functionen. 1867. p. 52.

* Studien über die Bessel'schen Functionen. 1868. p. 86 seq. 
oder:

$$
E^{n}(x)=\frac{1}{\Gamma\left(\frac{1}{2}\right) \Gamma\left(n+\frac{1}{2}\right)}\left(\frac{x}{2}\right)^{n} \int_{0}^{\pi} \cos (x \cos \varphi) \cdot \sin ^{y n} \varphi \log \sin ^{2} \varphi d \varphi
$$

$$
\begin{gathered}
E^{n}(x)=2\{\psi(2 n+1)-\psi(n+1)-\log 2\} J^{n}(x) \\
+\Sigma \frac{(-1)^{p}}{\Gamma(n+p+2) \Gamma(p+2)}\left(\frac{x}{2}\right)^{n+2 p+2}\{\psi(n+p+2)-\psi(n+1)\},
\end{gathered}
$$

gebracht, wo

$$
\psi(x)=\frac{\partial \log \Gamma(x)}{\partial x}
$$

und somit $\psi(1)$ der negativen Constanten des Integrallogarithmus $\psi(1)=-0,5772 \ldots$ gleich gesetzt wird*).

Dass die grosse Weitläufigkeit und geringe Durchsichtigkeit dieser Ausdrücke eine eingehende Untersuchung derselben verhindert, liegt auf der Hand. Durch grössere Einfachheit empfehlen sich die Integralformen und es hat $\mathrm{Hr}$. Heine $\mathrm{e}^{* *}$ ) eine zweite particuläre Lösung durch das Integral

$$
x^{n} \int_{0}^{\infty} e^{x i \cos \varphi i} \sin ^{2 n} \varphi i d \varphi
$$

dargestellt, analog der älteren Formel:

$$
J^{n}(x)=\frac{1}{\Gamma\left(\frac{1}{2}\right) \Gamma\left(n+\frac{1}{2}\right)}\left(\frac{x}{2}\right)^{n} \int_{0}^{\frac{\pi}{2}} e^{x i \cos \varphi} \sin ^{2 n} \varphi d \varphi .
$$

Diese Form erscheint bei Hrn. Heine aus der Theorie der Kugelfunctionen abgeleitet, indèm die Cylinderfunctionen als Grenzfälle von Kugelfunctionen angesehen werden können. Allgemeiner gesprochen, kann man $J^{n}(x)$ als Grenzfall einer hypergeometrischen Reihe ansehen, und zwar, wie Hr. Hansen ) bemerkt hat:

$$
J^{n}(x)=\left(\frac{x}{2}\right)^{n} \frac{1}{\Gamma(n+1)} F\left(\omega, \omega^{\prime},(n+1),-\frac{x^{2}}{4 \omega \omega^{\prime}}\right)
$$

wo $\omega, \omega^{\prime}$ zwei positive unendlich wachsende Grössen bedeuten und $F$ die hypergeometrische Reihe:

*) Ganss hat bekantlich $\frac{\partial \log \Pi(x)}{\partial x}=\Psi(x)$ gesetzt, wo $\Pi(x)=\Gamma(x+1)$. Um nun diese Function $\Psi$ mit der $\Gamma$-Function in bequeme Verbindong zu bringen, habe ich obige Form angesetat, so dass das $G$ a uุs s'sche $\Psi(x)$ mein $\psi(x+1)$ ist.

*) Crelle, Journ. t. 69. p. 140.

***) Abhandlung d. Sächsischen Ges. d. Wissensch. Mathem.-phys, Classe t. II. 1855. p. 252 . 


$$
F(\alpha, \beta, \gamma, x)=\frac{\Gamma(y)}{\Gamma(\alpha) \Gamma(\beta)} \sum_{p=0}^{\infty} \frac{\Gamma(\alpha+p) \Gamma(\beta+p)}{\Gamma(\gamma+p) \Gamma(p+1)} x^{p}
$$

darstellt. Es hat die Reihe für $J^{n}(x)$ ein Element, $(n+1)$, welches in dem berührten Falle eine positive ganze Zahl ist, und eine eingehende Untersuchung zeigt, dass in diesem Falle die Differentialgleichung jener Reihe als zweite particuläre Lösung nicht eine zweite hypergeometrische Reihe $F$ hat, sondern dass diese anderen Lösungen aus gewissen abgebrochenen hypergeometrischen Reihen $G(\alpha, \beta, \gamma, x)$ (ganzen Functionen von $x$ ) und neuen Reihen von dem Typus:

$$
\begin{gathered}
H(\alpha, \beta, \gamma, x)=\frac{1}{\Gamma(\alpha) \Gamma(\beta) \Gamma(\gamma-1)} \sum_{p=0}^{\infty} \frac{\Gamma(\alpha+p) \Gamma(\beta+p)}{\Gamma(\gamma+p) \Gamma(p+1)} x^{p} \times \\
\{\psi(\gamma+p)+\psi(p+1)-\psi(\alpha+p)-\psi(\beta+p)-\log x\}
\end{gathered}
$$

zasammengesetzt sind.

Es ist nicht meine Absicht, hier in eine allgemeine Theorie dieser anomalen, bisher nur sporadisch bemerkten Fälle einzugehen, was ich einer folgenden Abhandlung vorbehalte. Ich begnüge mich hier, die particuläre Lösung $Y$ in der neuen Form $H(\alpha, \beta, \gamma, x)$ von Reihen $\mathrm{zu}$ entwickeln und die, wie ich meine, natürlichste Ableitung derselben zu geben, die ihr Wesen vollständig aufzuklären geeignet ist.

In einigen weiteren Paragraphen habe ich dann die $\mathcal{J}$ und $Y$ als Integrale dargestellt and die Werthe sämmtlicher Integrale (auf reellen und complexen Wegen), die als Lösungen der Differentialgleichung angesehen werden können, vollständig untersucht, indem ich hier, wie in der ganzen Abhandlung sogleich die allgemeine Voraussetzung eines complexen Index $n$ und einer complexen Variabelen $x$ mache.

Am Schlusse habe ich die bisher nur theilweise bekannten semiconvergenten Entwickelungen der Cylinderfunctionen erster und zweiter Art aufgestellt, und ihnen eine eingehendere Discussion für complexe Werthe des Argumentes gegeben, die $\mathrm{zu}$ eigenthümlichen Resultaten geführt hat und vielleicht als erster Baustein zu einer allgemeinen Theorie der semiconvergenten Reihen für unbeschränkte Variabilität des Argumentes dienen kann; denn die eigentliche Natur dieser merkwürdigen Entwicklungen ist noch fast ganz unerkannt; und es dürfte erst die complexe functionentheorie ihr Wesen zur Erkenntniss bringen, ebenso als auch sie erst die wahre Natur der convergenten Potenzreihen aufgedeckt hat.

\section{§. 1.}

\section{Entwickelung von $Y$ in eine Reihe.}

Wenn $n$ eine positive ganze Lahl ist, so redueirt sich $J^{-n}(x)$ auf 
$J^{+n}(x)$; denn lässt man unter jener Voraussetzung eines ganzen positiven $n$ in:

$$
J^{-(n-\varepsilon)}(x)=\left(\frac{x}{2}\right)^{-(n-\varepsilon)} \sum_{p=0}^{\infty} \frac{(-1)^{p}}{\Gamma(-n+\varepsilon+p+1) \Gamma(p+1)}\left(\frac{x}{2}\right)^{2 p}
$$

die Grösse $\varepsilon$ zu Null abnehmen, so verschwinden alle Glieder der Reihe bis zu $p=n$, da $\Gamma(-n+p+1)$ so lange unendlich gross wird, als das Argument eine negative ganze Zahl oder Null ist. Setzt man in der zweiten Summe von $p=n$ an $p-n$ einem neuen Summationsbuchstaben $p$ gleich, so hat man:

$$
\begin{gathered}
J^{-(n-\varepsilon)}(x)=\left(\frac{x}{2}\right)^{-(n-8)} \sum_{p=0}^{n-1} \frac{(-1)^{p}}{\Gamma(-n+\varepsilon+p+1) \Gamma(p+1)}\left(\frac{x}{2}\right)^{2 p} \\
+(-1)^{n}\left(\frac{x}{2}\right)^{n+\varepsilon} \sum_{p=0}^{\infty} \frac{(-1)^{p}}{\Gamma(\varepsilon+p+1) \Gamma(n+p+1)}\left(\frac{x}{2}\right)^{2 p}
\end{gathered}
$$

und, wenn $\varepsilon=0$, fällt die erste Reihe ganz weg, die zweite verwandelt sich in $J^{+n}(x)$, so dass:

$$
J^{-n}(x)=(-1)^{n} J^{+n}(x) .
$$

Um aber auch in diesem Falle eine zweite particuläre Lösung zu erhalten, bemerken wir, dass, weil $(-1)^{n} J^{-(n-\varepsilon)}(x)-J^{n-\varepsilon}(x)$ mit abnehmendem $\varepsilon$ verschwindet, der Quotient

$$
\frac{(-1)^{n} J^{-(n-\varepsilon)}(x)-J^{n-\varepsilon}(x)}{\varepsilon}
$$

sich einer Grenze nähern wird, und da er im Allgemeinen, was auch $n$ und $\varepsilon$ seien, eine particuläre Lösung darstellt, auch im Falle eines ganzen $n$ und abnehmenden $\varepsilon$ eine solche liefern wird. Es ist leicht, den Grenzwerth jenes Quotienten darzustellen; denn setzt man vorstehende Reihen ein, so wird er:

$=\left(\frac{x}{2}\right)^{-(n-\varepsilon)} \sum_{p=0}^{n-1} \frac{(-1)^{p+n}}{\Gamma(p+1) \varepsilon \Gamma} \frac{1}{\Gamma(-n+\varepsilon+p+1)}\left(\frac{x}{2}\right)^{2 p}+\left(\frac{x}{2}\right)^{n} \sum_{p=0}^{\infty}(-1)^{p} \frac{f(z)}{\varepsilon}\left(\frac{x}{2}\right)$

wo $f(\varepsilon)$ den von $\varepsilon, p, x$ abhängenden Ausdruck repräsentirt:

$$
f(\varepsilon)=\frac{1}{\Gamma(n+p+1) \Gamma(p+\varepsilon+1)}\left(\frac{x}{2}\right) \cdot-\frac{1}{\Gamma(n+p-\varepsilon+1) \Gamma(p+1)}\left(\frac{x}{2}\right)^{-\varepsilon}
$$

Den Grenzwerth, dem sich das hier eingeführte $f(\varepsilon)$, dividirt durch $\varepsilon$, nähert, findet man leicht durch Differentiation des Zählers und Nenners nach $\varepsilon$ und nachherige Substitution $\varepsilon=0 \mathrm{zu}$ :

$$
\begin{aligned}
& \frac{1}{\Gamma(n+p+1) \Gamma(p+1)} 2 \log \frac{x}{2}-\frac{1}{\Gamma(n+p+1)} \frac{\Gamma^{\prime}(p+1)}{[\Gamma(p+1)]^{2}}-\frac{1}{\Gamma(p+1)} \frac{\Gamma^{\prime}(n+p+1)}{[\Gamma(n+p+1)]^{2}} \\
& =\frac{1}{\Gamma(n+p+1) \Gamma(p+1)}\left\{\log \left(\frac{x}{2}\right)^{2}-\psi(p+1)-\psi(n+p+1)\right\} .
\end{aligned}
$$


Die einfache Transformation von

$$
\frac{1}{\Gamma(-n+\varepsilon+p+1)}=\frac{1}{\pi} \Gamma(n-\varepsilon-p) \sin (-n+\varepsilon+p+1) \pi
$$

mittelst der Formel: $\Gamma(x) \Gamma(1-x) \sin x \pi=\pi$ liefert ferner den Grenzwerth von:

$$
\frac{1}{\varepsilon \Gamma(-n+\varepsilon+p+1)}=(-1)^{n+p+1} \Gamma(n-p)
$$

und somit schliesslich als zweite particuläre Lösung:

$$
\begin{gathered}
Y^{n}(x)=-\left(\frac{x}{2}\right)^{-n} \sum_{p=0}^{n-1} \frac{\Gamma(n-p)}{\Gamma(p+1)}\left(\frac{x}{2}\right)^{2 p} \\
+\left(\frac{x}{2}\right)^{n} \sum_{x=0}^{\infty} \frac{(-1)^{p}}{\Gamma(n+p+1) \Gamma(p+1)}\left(\frac{x}{2}\right)^{2 p}\left\{\log \left(\frac{x}{2}\right)^{2}-\psi(n+p+1)-\psi(p+1)\right\},
\end{gathered}
$$

welche sich von dem oben mit $\dot{Y}$ bezeichneten Ausdruck nur durch den Factor 2 und ein Vielfaches von $J^{n}(x)$ unterscheidet.

So hat $\operatorname{man}$ z. B. für $n=0$ :

$$
Y(x)=2 \sum_{p=0}^{\infty} \frac{(-1)^{p}}{[\Gamma(p+1)]^{2}}\left(\frac{x}{2}\right)^{2 p}\left\{\log \frac{x}{2}-\psi(p+1)\right\},
$$

in welcher Form schon früher Riemann*), jedoch ohne alle Ableitung, die Lösung in diesem speciellen Falle gegeben hat. Für $n=1$ findet man:

$$
Y^{1}(x)+\frac{2}{x}=
$$$$
=\frac{x}{2} \sum_{p=0}^{\infty} \frac{(-1)^{p}}{\Gamma(p+2) \Gamma(p+1)}\left(\frac{x}{2}\right)^{2 p}\left\{\log \left(\frac{x}{2}\right)^{2}-\psi(p+2)-\psi(p+1)\right\}
$$

u. s. w. Wir haben somit diese zweite Lösung entwickelt in der denkbareinfachsten Reihenform, die sich der der J function soweit, als nach der Natur der Sache möglich, anschliesst; während die oben citirten Formen der Hrn. Neumann und Lommel, $Y$ selbst nur entwickelt nach $J$ functionen aufzeigen.

Was übrigens die Convergenz dieser Reihen $Y$ betrifft, so ist der Quotient zweier aufeinander folgender Glieder:

$$
-\frac{1}{(n+p+1)(p+1)} \frac{x}{2} \frac{\log \left(\frac{x}{2}\right)^{2}-\psi(n+p+2)-\psi(p+2)}{\log \left(\frac{x}{2}\right)^{2}-\psi(n+p+1)-\psi(p+1)} .
$$

*) Poggendorft's Annalen. \&. 95. p. 135. 
Da num der asymptotische Werth von $\Gamma(q)$ mit unendlich wachsendem positivem $q$ bekanntlich

$$
e^{\left(q-\frac{1}{2}\right) \log q-q}
$$

der von $\psi(q)$ also: $\log q$ ist, so wird sich jener Quotient mit wachsendem $p$ der Grenze:

$$
-\frac{1}{(n+p+1)(p+1)} \frac{x}{2} \frac{\log (n+p+2)(p+2)}{\log (n+p+1)(p+1)}
$$

nähern, also unendlich abnehmen; und die Reihe $Y$ convergirt daher, wie die Reihe $J$, für jedes endliche complexe $x$.

Die Lösungen $\bar{Y}^{n}(x)$ für ein ganzes positives $n$ haben übrigens einen wesentlich anderen Charakter als die Cylinderfunctionen erster Art, die $J^{n}(x)$;-denn während $x^{-n} J^{+n}(x)$ in der ganzen Ebene der complexen $x$ eine eindeutige Function der Variabélen $x$ ist und $J^{n}(x)$ bei einem Umlaufe um $x=0$ den Factor $e^{2 n \pi i}$ annimmt, der im Falle eines positiven ganzen $n$ sich auf 1 reducirt, hat $Y^{n}(x)$ in $x=0$ einen solchen Verzweigungspunkt, dass bei seinem Umkreisen $Y^{n}(x)$ um $4 \pi i J^{n}(x)$ additiv zunimmt. Im Allgemeinen wird übrigens $Y^{n}(x)$ für $x=0$ unendlich, wie $x^{-n}$; für $n=0$ aber wird $Y(x)$ in $x=0$ logarithmisch unendlich werden.

Da die beiden Lösungen $J^{+n}(x)$ und $J^{-n}(x)$ für ganze $n$ zusammenfallen, wir aber in $Y^{n}(x)$ eine lineare Function beider gefunden haben, welche immer von ihnen verschieden bleibt, so kann es zweckmässig erscheinen, neben $J^{n}(x)$ noch für ein allgemeines $n$ als andere particuläre Lösung:

$$
Y^{n}(x)=2 \pi e^{n \pi i} \frac{J^{n}(x) \cdot \cos n \pi-J^{-n}(x)}{\sin 2 n \pi}
$$

einzuführen, die dann für den speciellen Fall eines ganzen $n$ in die obige Lösung übergeht. Auch werden unten die Summen:

$$
\begin{aligned}
\frac{J^{n}(x)-e^{n \pi i} J^{-n}(x)}{\sin 2 n \pi} & =\frac{1}{2 \pi} Y^{n}(x)-\frac{i}{2} \frac{e^{n \pi i}}{\cos n \pi} J^{n}(x) \\
e^{n \pi i} \frac{e^{n \pi i} J^{n}(x)-J^{-n}(x)}{\sin 2 n \pi} & =\frac{1}{2 \pi} Y^{n}(x)+\frac{i}{2} \frac{e^{n \pi i}}{\cos n \pi} J^{n}(x)
\end{aligned}
$$

als particuläre Lösungen auftreten.

$$
\text { \$. } 2 .
$$

\section{Uebersicht der Integralformen für die Cylinderfunetionen.}

Um die Differentialgleichung, die wir der Bequemlickkeit wegèn:

$$
\frac{\partial x^{2 n+1} \frac{\partial x^{-n} R}{\partial x}}{\partial x}=-x^{n+1} R
$$


schreiben, durch Integrale aufzulösen, setzen wir, entsprechend dem bekannten Integrale:

allgemein :

$$
R=x^{n} \int_{-1}^{+1} e^{x t i}\left(t^{2}-1\right)^{n-\frac{1}{2}} d t
$$

$$
R=x^{n} \int_{a}^{b} e^{x t i}\left(t^{2}-1\right)^{n-\frac{1}{2}} d t
$$

und arhalten so auf bekannte Weise als Bedingung für die Grenzen:

$$
\int_{a}^{b} e^{x t i}\left(t^{2}-1\right)^{n+\frac{1}{2}}=0
$$

I. Unter der Voraussetzung eines complexen $n$ und $x$ mit positiven reellen Theilen wird $e^{x t i}\left(t^{2}-1\right)^{n+\frac{1}{2}}$ für $t=+1,-1$ und für ein $t$ mit nnendlich grossem imaginären Theile, $t=\infty i$, verschwinden. Da aber das $\int e^{x t i}\left(t^{2}-1\right)^{n-\frac{1}{2}} d t$ unter diesen Voraussetzungen des Werthes von $x$, über einen Theil des oberhalb der reellen Axe gezogenen unendlichen Kreises genommen, verschwindet, so können wir statt $t=\infty i$ auch $t=k+\infty i$ setzen. Wir erhalten in diesem Sinne als particuläre Integrale:

$$
\begin{aligned}
& x^{n} \int_{-1}^{1} e^{x t i}\left(t^{2}-1\right)^{n-\frac{1}{2}} d t, \\
& x^{n} \int_{+1}^{+\infty i} e^{x t i}\left(t^{2}-1\right)^{n-\frac{1}{2}} d t, \\
& x^{n} \int_{-1}^{+\infty i} e^{x t i}\left(t^{2}-1\right)^{n-\frac{1}{2}} d t,
\end{aligned}
$$

zwischen denen die lineare Relation:

$$
\int_{-1}^{+1}+\int_{+1}^{+\infty i}+\int_{+\infty i}^{+1}=0
$$

besteht, wenn die Potenzen in entsprechender Weise bestimmt werden. In allen diesen Integralen hat man von einer der Grenzen anf einer einfachen Linie, die keinen Verzweigungspunkt $t= \pm 1 \mathrm{um}$ kreist, zu der anderen Grẹnze, und zwar am einfachsten geradlinig zu gehen. Eine andere Classe von Integralen entsteht, wenn man von einem der Grenzṕnkte aus zu ihm zurïckkehrt, nachdem man unterdessen einen der Verzweigungspunkte umkreist hat. So hat man als particuläres Integral

$$
x^{n} \int_{+1}^{+1} e^{x t i}\left(t^{2}-1\right)^{x-\frac{1}{2}} d t
$$

wenn man dabei von +1 ausgehend, den Punkt $t=-1$ amkreist and so wieder zn $t=+1$ zurüekkehrt. Ebenso ist: 


$$
x^{n} \int_{-1}^{-1} e^{x t i}\left(t^{2}-1\right)^{n-\frac{1}{2}} d t
$$

wenn man auf dem Wege den Punkt $t=+1$ umkreist, ein particuläres Integral. Das

$$
x^{n} \int_{+\infty i}^{+\infty i} e^{x t i}\left(t^{2}-1\right)^{n-\frac{1}{2}} d t
$$

kann auf drei versehiedene Weisen genommen werden, entweder, indem man von $+\infty i$ ansgehend, den Punkt $t=+1$ allein, oder $t=-1$ allein, oder beide gleichzeitig umkreist.

Es leuchtet ein, dass alle diese Integrale lineare Functionen zweier particulärer Integrale, z. B. $J^{+n}(x)$ und $J^{-n}(x)$ oder $J^{n}(x)$ und $Y^{n}(x)$ sein müssen, und wir werden in $\$ .3$ und 4 diese Reduction thatsächlich ausfiihren. Zuvor aber mag dieses System von Integralen noch weiter verrollständigt werden.

Bei der Symmetrie der ursprünglichen Differentialgleichung nach $+n$ und $-n$, hat man auch

$$
x^{-n} \int_{a}^{b} e^{x t i}\left(t^{2}-1\right)^{-n-\frac{1}{2}} d t
$$

als particuläre Integrale anzusenen, wo

$$
\int_{a}^{b} e^{x l i}\left(t^{2}-1\right)^{-n+\frac{1}{2}}=0 .
$$

Wenn nun die bisherigen Annahmen festgehalten werden, wonach $x$ und $n$ positive reelle Theile haben, so sind von diesen Integralen nur die drei in:

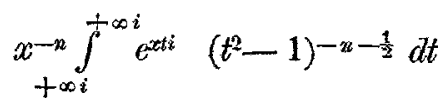

enthaltenen zu gebrauchen: wenn man entweder um jeden der Verzweigungspunkte $t= \pm 1$ einzeln oder um beide zusammen integrirt. In den anderen wird dagegen die Function unter dem Integralzeichen an den Grenzen im Allgemeinen so unendlich, dass die Integrale ihre Bedeutung verlieren. Wir haben somit in diesem Falle 11 brauchbare particuläre Integrale.

Wenn dagegen $n$ einen negativen reellen Theil hat, so sind letztere Integrale die, welche man benutzen wird; oder einfacher: Es treten dann 11 Integrale auf, welche man aus den aufgestellten (1) bis (7) durch Vertauschung von $+n$ mit $-n$ erhält.

Zwischen diesen beiden Fällen findet indess ein Uebergang statt; denn wenn der reelle Theil von $n$ zwisehen $\pm \frac{1}{2}$ liegt, so verschwindet $\left(t^{2}-1\right)^{+n+\frac{1}{2}}$ in den Punkten $t= \pm 1$ immer, und es sind dann (1) bis (6) und die ans ihnen durch Vertausehung von $+n$ mit $-n$ 
erhaltenen, also im Ganzen 16, sämmtlich brauchbare particuläre Integrale.

II. Bisher ist $x$ mit positivem reellen Theile vorausgesetzt worden. Nehmen wir, indem wir zunächst die Voraussetzung eines $n$ mit positivem reellen Theile beibehalten, jetzt $x$ mit negativem reellen Theile an, so behalten die Integrale (1), (4), (5) ihre Bedeutung bei. Da aber $e^{x t i}$ jetzt nicht für $t=+\infty i$, sondern für $t=-\infty i$ verschwindet, so hat man die übrigen Integrale (2), (3), (6), (7) durch entsprechende Integrale nach $t=-\infty i$ zu ersetzen, $d$. h. in das unendlich negative Imaginäre hinaus za integriren, wobei es gleichgültig ist, in welcher Weise man den reelen Theil des $t$ bestimmt.

Wie man zu verfahren hat, wenn unter derselben Voraussetzung für $x, n$ einen negativen oder einen zwischen $\pm \frac{1}{2}$ liegenden reellen Theil hat, bedarf jetzt weiter keiner Auseinandersetzung.

III. Ist endlich $x$ rein positiv imaginär (sein reeller Theil Null), so hat man an (1), (4), (5) nichts zu ändern; die Integrale $(2),(3),(6),(7)$ müssen aber jetzt nach $t=+\infty$ erstreckt werden, weil $e^{x t i}$ dort verschwindet; und zwar gelten diese unter der Voraussetzung eines positiven reellen Theiles ron $n$. Doch mag noch bemerkt werden, dass in diesem Falle die drei in (7) enthaltenen Integrale mit den dortigen Grenzen $t=\infty_{i} i$ beibehalten werden können, unter der Bedingung jedoch, dass $t=k+\infty i$ gesetzt, der reelle Theil $k$ nicht unendlich in das Negative wächst. Denn unter dieser Bedingung bleibt $e^{x t i}$ an der obéren Grenze endlich und $\left(1-t^{2}\right)^{-n-\frac{1}{2}}$ wird die ausreichende unendliche Decrescenz der Function an der oberen Grenze des Integrales herstellen. Ebenso würde man in diesem Falle $t=k-\infty i$ als die obere Grenze ansehen können.

Wie man im Falle eines $n$, das einen negativen oder zwischen It $\frac{1}{2}$ "gelegenen reellen Theil besitzt, die Integrale auszuwählen hat, und dass man, wenn

IV, $x$ eine rein negativ imaginäre Grösse ist, $t=-\infty$ im Allgemeinen als obere Grenze anzusehen hat, leuchtet nach Vorstehendem genügend ein. -

Wir haben so das vollständige System der Integrale betrachtet, welche als particuläre Lösungen unserer Differentialgleichung angesehen werden können: Je nach der Beschaffenheit des $x$ und $n$ sind bald diese, bald jene Formen zu gebrauchen: Mit $x$ ändert sich die Art und Weise, wie man in diesen Integralen die Variabeln in's Unendliche wachsen lassen muss. Es kann dies in der Form zasammengefasst werden, dass $t$ so ' wachsen soll, dass $x t i=-\infty$ ist, also $t=\frac{1}{x} \infty i$. Dies führt darauf, $x t i=-u$ als neve Veränderliche einzuführen, and so die particulären Integrale in der Form: 


$$
x^{-n} \int e^{-u}\left(u^{2}+x^{2}\right)^{n-\frac{1}{2}} d u
$$

anzunehmen. An Stelle der Grenzen $t= \pm 1$ treten jetzt $u= \pm x i$ auf, and man hat für den unendlichen Werth der Integrationsvariabelen jetzt in jedem Falle $u=+\infty$ zu setzen (oder allgemeiner $u=\infty+k i$, wo $k$ eine ganz beliebige, endliche oder unendlich wachsende reelle Grösse ist). Die drei Integrale:

$$
x^{-n} \int_{\infty}^{\infty} e^{-u}\left(u^{2}+x^{2}\right)^{+n-\frac{1}{2}} d u
$$

wo man um $u=+x i$ allein, oder um $u=-x i$ allein, oder um diese beiden Verzweigungspunkte gleichzeitig zu integriren hat; wie die weiteren drei in:

$$
x^{n} \int_{\infty}^{\infty} e^{-u}\left(u^{2}+x^{2}\right)^{-n-\frac{1}{2}} d u
$$

enthaltenen Integrale gelten jetzt unverändert für jedes $n$ und $x$.

Die Integrale:

$$
\int_{-x i}^{+x i}, \int_{+x i}^{\infty}, \int_{-x i}^{\infty}, \int_{+x i}^{+x i}, \int_{-x i}^{x i}
$$

der Function

$$
x^{-n} e^{-u}\left(u^{2}+x^{2}\right)^{n-1}
$$

gelten jetzt, wenn $n$ einen positiven reellen Theil hat, unbedingt für jedes $x$. Wenn aber $n$ einen negativen rellen Theil hat, so treten an ihre Stelle dieselben Integrale der anderen Function:

$$
x^{n} e^{-u}\left(u^{2}+x^{2}\right)^{-n-\frac{1}{2}},
$$

and wenn der reelle Theil von $n$ zwischen $\pm \frac{1}{2}$ liegt, so kann man sämmtliche Integrale benutzen.

Trotz der etwas allgemeineren Gültigkeit, welche den Integralen der letzteren Form zukommt, habe ich es doch vorgezogen, die folgenden Entwickelungen an die frühere und ältere Form anzuschliessen.

\section{§. 3.}

\section{Die Integrale für die Cylinderfunctionen erster Art.}

Um nnn die vorstehenden Integrale za entwickeln and sie so anf $J^{n}(x), J^{-n}(x), Y^{n}(x)$ reduciren zu können, hat man zunächst festzusetzen, wie man die vieldentige Potenz $\left(t^{2}-1\right)^{n-\frac{1}{2}}$ oder überhanpt $\left(t^{2}-1\right)^{q}$ in der Ebene der complexen $t$ bestimmen solle. Da

$$
\left(t^{2}-1\right)^{q}=(t-1)^{q}(t+1)^{q}
$$


so sieht man, dass diese Function die drei Verzweigungspunkte $-1,+1, \infty$ hat. Läuft $\operatorname{man}$ um $t=+1$ oder $t=-1$ herum, und kehrt wieder zum Ausgangspunkte zurïck, so hat die Potenz den Factor $e^{2 \eta \pi i}$ angenommen, und wenn man den Punkt $t=\infty$ umkreist, den Factor $e^{4} \pi_{2} ;$ wenn in beiden Fällen in der positiven Richtung (d. h. bei gewöhnlicher Lage der reellen und imaginären Axe gegen einander, umgekehrt wie der Zeiger der Uhr) die etwa kreisförmige Curve durchlaufen wird. Die Potenz wird demnach eindeutig sein, so lange man nicht einen dieser Punkte umkreist, also innerhalb der Ebene, wenn man sie sich in einer alle drei Verzweigungspunkte verbindenden, einfachen, keinen Knoten bildenden Linie zerschnitten denkt.

Nur in dem Falle, dass $2 q[=2 n-1]$ eine ganze Zahl ist, wird die Zerschneidung eine andere. Ist $2 q$ eine gerade [also $\left(n-\frac{1}{2}\right)$ eine ganze] Zahl, so wird die Potenz überhaupt nicht vieldeutig. Ist aber $2 q$ eine ungerade [ $n$ eine ganze] Zahl, so ist $t=\infty$ kein Verzweigungspunkt mehr und die Ebene ist nur zwischen -1 und +1 zerschnitten zu denken. Doch bedürfen diese speciellen Fälle im Folgenden keiner speciellen Behandlung, insofern sie von selbst in den allgemeinen Fall eingeschlossen - sind.

Um num im Allgemeinen $\left(t^{2}-1\right)^{q}$ zu bestimmen, betrachten wir seine Factoren. Soll der erste derselben $(t-1)^{q}$ eindeutig sein, so denken wir uns die Ebene von $t==1$ in einer in das Unendliche laufenden Linie zerschnitten*), und setzen dann:

$$
t-1=r e^{\varphi i},(t-1)^{q}=r^{q} e^{\varphi q i},
$$

$\varphi$ awischen 0 und $2 \pi$ liegen soll, und

$$
r^{q}=e^{q \log r}
$$

wo $\log r$ der reelle Logarithmus dieser positiven Grösse, also $r^{q}$ für ein reelles $q$ der positive reelle Werth dieser Potenz ist. Dann ist für Werthe von $t$ in der reellen Axe zwischen -1 und +1 :

$$
t-1=r e^{\pi i},(t-1)^{q}=r^{q} e^{q \pi i}
$$

*) In Betreff der Bedeutang einer solchen Zerschneidung mag folgendes bemerkt werden: Geschieht die Zerschneidung in der reellen Axe von 1 bis $\infty$, so bedeutet dies nichts anderes als: Es soll in $t-1=r e^{\varphi^{i}}$, worin $\varphi$ bekanntlich um ganze Vielfache von $2 \pi i$ unbestimmt ist, die Variabilität von $\varphi$ zwischen den Grenzen 0 und $2 \pi$ eingeschlossen sein. Wird in der Linie von $t=1$ bis $t=1+\infty i$ zerschnitten, so wird $\varphi$ zwischen den Grenzen $+\frac{\pi}{2}$ und $5 \frac{\pi}{2}$ anznnehmen sein, oder etwa auch zwischen $-3 \frac{\pi}{2}$ and $+\frac{\pi}{2}$ and man wird diese beiden Annahmen dadurch unterscheiden können, dass man sagt, es solle die Potenz genommen werden, welche für $t=2$ entweder $=1$ ist, wo dann $t-1=e^{0 i},(t-1)^{q}=1^{q}=1$ zu setzen wäre, oder im anderen Falle, wo $t-1=e^{2 \pi i}$ gesetzt ist, die Potenz, welche sich für $t=2$ anf $e^{2 q x i}$ reducirt. 
Fermer nehmen wir, um die Potenzen von $(t+1)$ eindeutig zu machen, die Ebene von -1 bis +1 in der reellen Axe und von 1 bis in's Unendliche in der schon vorhin festgesetzten Linie zerschnitten an, und setzen, indem wir $2 \pi>\varphi^{\prime}>0$ annehmen:

$$
t+1=r^{\prime} e^{\varphi^{\prime} i},(t+1)^{q}=r^{\prime q} e^{q\left(\varphi^{\prime}-2 \pi\right) i}
$$

so dass oberhalb der reellen Axe zwischen $t=-1$ und +1

und unterhalb

$$
t+1=r^{\prime} \quad(t+1)^{q}=r^{q} e^{-2 q \pi i}
$$

za nehmen ist.

$$
t+1=r e^{2 \pi i} \quad(t+1)^{q}=r^{q}
$$

Dureh Superposition finden wir dann in demselben Intervalle, oberhalb der reellen Axe .

$$
(t+1)^{q}(t-1)^{q}=\left(r r^{\prime}\right)^{q} e^{-q \pi i},\left(t^{2}-1\right)^{q}=\left(1-t^{2}\right)^{q} e^{-q \pi i}
$$

und anterhalb

(II) $\quad(t+1)^{q}(t-1)^{q}=\left(r r^{\prime}\right)^{q} e^{t q \pi i},\left(t^{2}-1\right)^{q}=\left(1-t^{2}\right)^{q} e^{+q \pi i}$,

wenn wir mit

$$
\left(r r^{\prime}\right)^{q}=\left(1-t^{2}\right)^{q}
$$

diejenige Potenz von $\left(1-t^{2}\right)$ bezeichnen, welche sich aus

$$
\left(1-t^{2}\right)^{q}=e^{q \log (1-n)}
$$

für den reellen Logarithmus dieser positiven Grösse $\left(1-t^{2}\right)$ ergiebt, d. h. diejenige, welche die Entwickelung nach dem binomischen Lehrsatze:

$$
\left(1-t^{2}\right)^{q}=\Gamma(q+1) \sum_{p=0}^{\infty} \frac{(-1)^{p}}{\Gamma(q-p+1) \Gamma(p+1)} t^{2 q}
$$

liefert, die sich für $t=0$ auf +1 reducirt. -

Wir betraehten nun das Integral

$$
\int_{-1}^{+1} e^{x t i}\left(t^{2}-1\right)^{x-\frac{1}{2}} d t
$$

fig. 1.

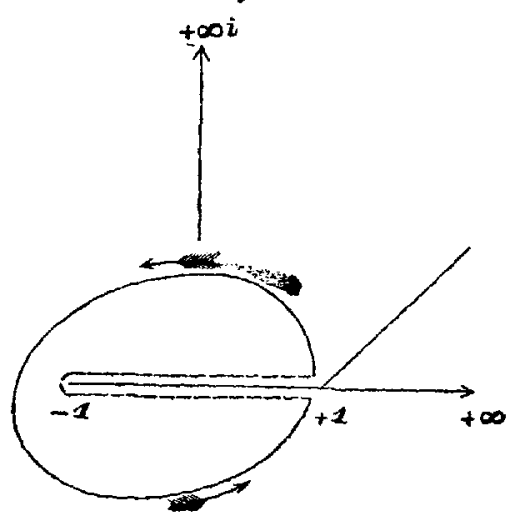

von +1 oberhalb der Zerschneidung und um -1 herum nach +1 noterhalb der Zerschneidung gefïhrt, auf einer Curve, wie' sie etwa in Fig. 1. ausgezogen ist. Dann kann man unter der nothwendigen Voraussetzang eines $n$ mit positivem reellen Theile das Integral auf eine Curve zusammenziehen, wie sie in Fig. 1. punktirt gezogen ist; und zeifallt man das Integral in eines $\int_{+1}^{-1}$ oberhalb des Schnittes und eines $\int_{-1}^{+1}$ un- 
terhalb desselben, beachtet ferner die in (I), (II) angegebenen Zeichen der Potenzen in diesen, so findet man obiges Integral

$$
\begin{array}{r}
=e^{-\pi\left(n-\frac{1}{2}\right) i} \int_{+1}^{-1} e^{x t i}\left(1-t^{2}\right)^{n-\frac{1}{2}} d t+e^{+\pi\left(n-\frac{1}{2}\right) i} \int_{-1}^{+1-} e^{x t i}\left(1-t^{2}\right)^{n-\frac{1}{2}} d t \\
=-2 i \cos n \pi \int_{-1}^{+1} e^{x t i}\left(1-t^{2}\right)^{n-\frac{1}{2}} d t .
\end{array}
$$

Reducirt man nun letzteres $\int_{-1}^{+1}$ auf $\int_{0}^{1}$, entwickelt den so entstehenden $\cos x t$ nach Potenzen von $x t$, integrirt mittels der bekannten Formel für die Euler'schen Integrale erster Art und wendet die auch im Folgenden häufig benutzten Formeln:

$$
\frac{\Gamma(2 p+1)}{\Gamma\left(p+\frac{1}{2}\right)}=2^{2 p} \frac{\Gamma(p+1)}{\Gamma\left(\frac{1}{2}\right)}, \Gamma\left(\frac{1}{2}+n\right) \Gamma\left(\frac{1}{2}-n\right)=\frac{\pi}{\cos n \pi}
$$

an, so findet man

$$
\int_{+1}^{+1} e^{x f i}\left(t^{2}-1\right)^{n-\frac{1}{2}} d t=-2 \pi i \frac{\Gamma\left(\frac{1}{2}\right)}{\Gamma\left(\frac{1}{2}-x\right)} \sum_{p=0}^{\infty} \frac{(-1)^{p}}{\Gamma(n+p+1) \Gamma(p+1)}\left(\frac{x}{2}\right)^{2 p} ;
$$

also nach dem Werthe letzterer Reihe:

$$
J^{n}(x)=\frac{i}{2 \pi} \frac{\Gamma\left(\frac{1}{2}-n\right)}{\Gamma\left(\frac{1}{2}\right)}\left(\frac{x}{2}\right)^{n} \int_{+1}^{+1} e^{x f i}\left(t^{2}-1\right)^{n-\frac{1}{2}} d t
$$

oder auch

$$
J^{n}(x)=\frac{1}{\Gamma\left(\frac{1}{2}+n\right) \Gamma\left(\frac{1}{2}\right)}\left(\frac{x}{2}\right)^{n} \int_{-1}^{+1} e^{x t i}\left(1-t^{2}\right)^{n-\frac{1}{2}} d t
$$

womit die Werthe der Integrale (1) und (4) des §. 2. gefunden sind. Auch (5) desselben §. liesse sich so ohne Schwierigkeiten finden, wenn man eine andere Zerschneidung einführte. -

Um weiter das Integral

$$
\int_{+\infty i}^{+\infty i} e^{x t i}\left(t^{2}-1\right)^{n-\frac{1}{2}} d t
$$

unter der Voraussetzung eines complexen $x$ mit positivem reellen Theile zu behandeln, denken wir uns den von +1 in's Unendliche gehenden Schnitt, dessen specielle Lage bisher willkürlich gelassen war, von +1 nach $+\infty i$, oder etwa geradlinig und der imaginären Axe parallel nach $1+\infty i$ ausgeführt und bestimmen die Potenzen beiderseits nach obigen Regeln (I), (II), indem wir in $t-1=r e^{\varphi i}$ die Variabilität von $\varphi$ zwischen den Grenzen $+\frac{\pi}{2}$ und $5 \frac{\pi}{2}$

Fig. 2.

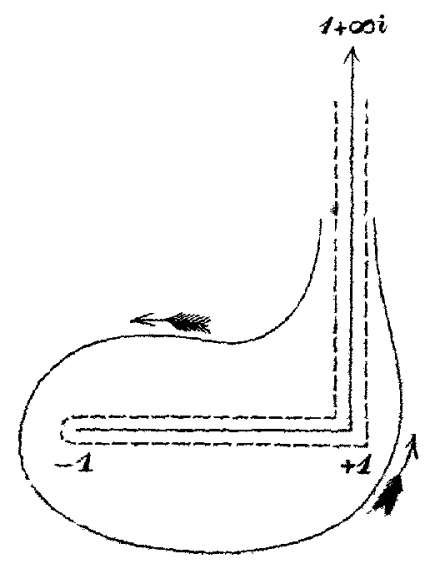


nehmen. Wir wollen dann das Integral von der linken Seite dieses Schnittes um -1 und +1 herum, ohne an den Schnitt zu treffen, auf der anderen, rechten Seite des Schnittes wieder hinaus nach $1+\infty i$, in einer Curve erstrecken, wie sie etwa in Fig. 2. dargestellt ist.

Um dann dies Integral nach aufsteigenden Potenzen von $x$ zu entwickeln, müssen wir zuvor

$$
\left(t^{2}-1\right)^{n-\frac{1}{2}}=1^{n-\frac{1}{2}} t^{2 n-1}\left(1-\frac{1}{t^{2}}\right)^{n-\frac{1}{2}}
$$

setzen und

$$
\left(1-\frac{1}{t^{2}}\right)^{n-\frac{1}{2}}=\Gamma\left(n+\frac{1}{2}\right) \sum_{p=0}^{\infty} \frac{(-1) p}{\Gamma\left(n-p+\frac{1}{2}\right) \Gamma(p+1)} \frac{1}{t^{2 p}}
$$

nach dem binomischen Lehrsatze entwickeln. Dies setzt aber voraus, dass wir mit unserer Integrationscurve immer ausserhalb des mit dem Radius 1 um $t=0$ beschriebenen Kreises bleiben, an welchem die Convergenz dieser Reihe aufhört. Um aber ausserhalb dieses Convergenzkreises die Gleichung:

$$
\left(t^{2}-1\right)^{n-\frac{1}{2}}=1^{n-\frac{1}{2}} t^{2 n-1}\left\{1-\left(n-\frac{1}{2}\right) \frac{1}{t^{2}}+\cdots\right\}
$$

behaupten $2 u$ können, müssen wir auch $t^{2 n-1}$ in der von 0 über 1 nach $1+\infty i$ zerschnittenen Ebene zu

$$
t=r^{\prime \prime} e^{\varphi^{\prime \prime} i}, t^{2 n-1}=r^{\prime \prime 2 n-1} e^{(2 n-1) \varphi^{\prime \prime} i}
$$

bestimmt denken; dann ist in der so zerschnittenen Ebene weder die linke, noch die rechte Seite vieldentig und es werden beide in allen Punkten übereinstimmen, wenn $1^{n-\frac{1}{2}}$ passend, d. h. so bestimmt ist, dass beide Seiten für Einen Punkt übereinstimmen. Wählen wir dazu einen Punkt auf der negativen reellen Axe links von -1 , so ist in diesem nach Obigem:

$$
(t-1)^{n-\frac{1}{2}}=r^{n-\frac{t}{2}} e^{t \pi\left(n-\frac{1}{2}\right) i},(t+1)^{n-\frac{1}{2}}=r^{n-\frac{1}{2}} e^{-\pi\left(n-\frac{1}{2}\right) i}
$$

also:

$$
\left(t^{2}-1\right)^{n-\frac{1}{2}}=\left(r r^{n}\right)^{n-\frac{1}{2}}
$$

und wird, wenn sich $t$ nach $-\infty$ entfernt, und $n$ eine positive reelle Zahl ist, in das positiv Unendliche wachsen. Gleichzeitig wird

$$
t^{2 n-1}=r^{\prime 22-1} e^{(2 n-1) \pi i}
$$

and soll die rechte Seite ebenfalls in's positiv Unendliche wachsen, so muss

$$
1^{x-\frac{1}{2}}=e^{-(2 n-1) x i}
$$

gesetat werden; so dass man:

$$
\left(t^{2}-1\right)^{n-1}=e^{-(2 x-1) \pi i} \Gamma\left(n+\frac{1}{2}\right) \sum_{x=0}^{\infty} \frac{(-1)^{p}}{\Gamma(n-p+1) \Gamma(p+1)} t^{2 n-2 p-1}
$$

zu setzen hat. Nach diesen Vorbereitungen hat man dann: 
$\int_{\infty i}^{\infty i} e^{x t i}\left(t^{2}-1\right)^{n-\frac{1}{2}} d t=e^{-(2 n-1) \pi i} \Gamma\left(n+\frac{1}{2}\right) \sum_{p=0}^{\infty} \frac{(-1)^{p}}{\Gamma\left(n-p+\frac{1}{2}\right) \Gamma(p+1)} \int_{\infty i}^{\infty i} e^{x t i} t^{2 n-2 p-1} d t$

Es handelt sich nun um die Ermittelung dieser Integrale:

$$
\int_{\infty i}^{\infty i t x^{x i}} t^{q} d t
$$

in denen $t^{q}$ die einzige vieldeutige Grösse ist, so dass man die Zerschneidung sich jetzt nur von 0 nach $\infty i$ und zwar, der Einfachheit wegen, auch direct in der imaginären positiven Axe vorgenommen denken kann. Es ist dann in

$$
t=r^{\prime \prime} e^{\varphi^{\prime \prime i}} \quad, \quad t^{q}=r^{\prime \prime q} e^{q \varphi^{\prime \prime} i}
$$

die Variabilität des $\varphi^{\prime \prime}$ auf die Werthe von $+\frac{\pi}{2}$ bis $5 \frac{\pi}{2}$ beschränkt.

Führen wir jetzt die neue Variabele $u$ durch $t=u i$ ein, so ist die neue $u$ Ebene jetzt von 0 bis $+\infty$ auf der positiven reellen Axe. zu zerschneiden, und bestimmen wir dem entsprechend

so ist

$$
u=r e^{p i}, u^{q}=r^{q} e^{q \varphi} \text {; }
$$

$$
t^{q}=i^{q} u^{q}
$$

eine bestimmte Gleichung, wenn $i^{q}$ entsprechend bestimmt wird. Nun ist für $t=-1, t^{q}=e^{q \pi i}$, und $u=+i=e^{\frac{\pi}{2} i}$ also $u^{q}=e^{q \frac{\pi}{2} i}$ und somit, wenn die Gleichheit beider Seiten hergestellt werden soll, $i^{q}=e^{q \frac{\pi}{2} i}$, also hat man:

$$
\int_{\infty}^{\infty i} e^{x t i} t^{q} d t=i e^{q \frac{\pi}{2} i} \int_{\infty}^{\infty} e^{-u x} u^{q} d u \text {. }
$$

Letztere Integrale aber lassen sich durch die $\Gamma$ function ausdrücken, was auch $q$ sei; und zwar folgendermassen:

Das Integral

$$
\int_{\infty}^{\infty} e^{-u x} u^{q} d u
$$

Fig. 3.

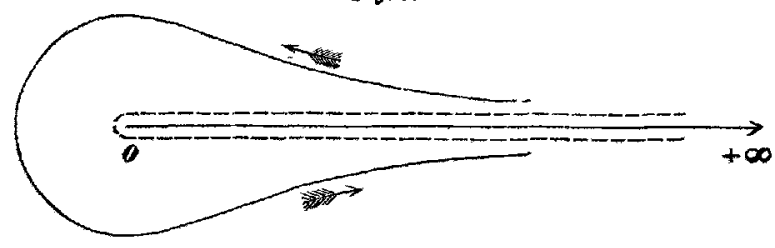

über den in Fig. 3. ansgezogenen Weg ausgedehnt, lässt sich, wenn $q$ eine complexe Zahl mit positivem reellen Theile ist, anf eine den Sehnitt von 0 bis $\infty$ anmittelbar umschliessende Linie (s. Fig. 3.) za. 
zusammenziehen, und beachtet man die Vorzeichen, welche $t^{q}$ oberund unterhalb des Schnittes annimmt, so findet man durch Zeriegung $\operatorname{des} \int_{\infty}^{\infty}$ in $\int_{\infty}^{0}$ oberhalb und $\int_{0}^{\infty}$ unterhalb:

$\int_{\infty}^{\infty} e^{-u x} u^{q} d u=\int_{\infty}^{0} e^{-x r} r^{q} d r+e^{2 q \pi i} \int_{0}^{\infty} e^{-x r} r^{q} d r=2 i e^{q \pi i} \sin q \pi \int_{0}^{\infty} e^{-x r} r^{q} d r$,

wo $r^{q}$ die Potenz ist, welche in der bekannten Definitionsgleichung von $\Gamma(q)$ für complexe $q$ mit einem reellen Theile, der grösser als -1 ist, nämlich in:

$$
\int_{0}^{\infty} e^{-x r} r d r=\frac{\Gamma(q+1)}{x q+1}
$$

erscheint. In dieser Gleiehung ist diejenige Potenz von $x^{q+1}$ zu nehmen, welche sich für $x=1$ auf +1 reducirt und man hat danach endlich:

$$
\int_{\infty}^{\infty} e^{-u x} u^{q} d u=-\frac{2 \pi i}{\Gamma(-q)} \frac{e q \pi i}{x q+1} .
$$

Da nun beide Seiten dieser Gleichung, auch wenn $q$ in seinem reellen Theile unter -1 abnimmt, stetig bleiben, wenn nur die Integrationscurve den kritischen Punkt $t=0$ in endlicher Entfernuug umläuft, so.gilt sie auch für solche $q$ und ist unter der Voraussetzung eines positiven reellen Theiles von $x$ für alle complexen $q$ gültig*).

Wird alles dies zusammengenommen, so findet man unser obiges Integral :

$$
\int_{\infty i}^{\infty i} e^{x t i}\left(t^{2}-1\right)^{n-\frac{1}{2}} d t=-2 \pi i e^{n \pi i} \Gamma\left(n+\frac{1}{2}\right) \Sigma \frac{x^{2 p-2 n}}{\Gamma\left(n-p+\frac{1}{2}\right) \Gamma(2 p-2 n+1) \Gamma(p+1)},
$$

und nach einfachen Transformationen erhält man rechts die Reihe für $J^{-n}(x)$, so dass:

$$
J^{-n}(x)=\frac{i}{2 \pi} e^{-n \pi i \frac{\Gamma\left(\frac{1}{2}-n\right)}{\Gamma\left(\frac{1}{2}\right)}}\left(\frac{x}{2}\right)^{n} \int_{\infty i}^{\infty i} e^{x t i}\left(t^{2}-1\right)^{n-\frac{1}{2}} d t .
$$

Diese Gleichung gilt für jedes $n$, da das Integral rechter Hand für alle $n$ seine Bedeutung beibehält; sie kann also arch zur Ermittelung des Integrales in Anspruch genommen werden, das aus diesem durch Vertauschung von $n$ mit $(-n)$ entsteht:

$$
J^{+n}(x)=\frac{i}{2 \pi} e^{+n \pi i} \frac{\Gamma\left(\frac{1}{2}+n\right)}{\Gamma\left(\frac{1}{2}\right)}\left(\frac{x}{2}\right)^{-n} \int_{\infty i}^{\infty i} e^{z i i} \frac{d t}{\left(t^{2}-1\right)^{n+1}} .
$$

*) Diese Formel and die daraus abzuleitenden Integrale für $\Gamma(q)$ habe ich bereits in der Dissertation: „Die Enler'schen Integrale bei unbeschränkter Variabilität des Argumentes". (Leipzig 1863) und im Anszage in Schlömilchs Journal t. IX. p. 8 gegeben. 


$$
\text { ร. } 4 .
$$

\section{Die übrigen Integrale der Differentialgleichung.}

Wenn in dem Integral $\left(6^{\mathrm{a}}\right)$ des vorigen $\$$. $n$ einen positiven reellen Theil hat, so ist man berechtigt, den Integrationsweg unendlich dicht an den Schnitt zusammenzuziehen, wie dies in Fig. 2. durch die punctirte Linie angedeutet ist. In $\mathrm{dem}$ speciellen Falle, dass $n$ eine positive ganze Zahl ist, wird die Potenz $\left(t^{2}-1\right)^{n-\frac{1}{2}}$ zu beiden Seiten der Linie von 1 bis $1+\infty i$ denselben Werth annehmen und sich daher $\int_{\infty i}^{\infty i} \operatorname{auf} \int_{+1}^{+1}$ reduciren, so dass man aus (4) und $\left(6^{a}\right)$

$$
J^{-n}(x)=e^{-n \pi i} J^{n}(x)
$$

erhält, eine schon früher bemerkte Relation. Man kann jedoch aus $\left(6^{a}\right)$ leicht ein Integral ableiten, welches immer von $J^{n}(x)$ verschieden bleibt:

Man hat nämlich, wenn $n$ einen positiven reellen Theil besitzt:

$$
\int_{\infty i}^{\infty i}=\int_{\infty i}^{+1}+\int_{+1}^{+1}+\int_{+1}^{\infty i}
$$

und da $\int_{f}^{\infty i}$ rechter Seits an dem Schnitte entlang zu nehmen ist, wo $\left(t^{2}-1\right)^{n-\frac{1}{2}}$ den Factor $e^{4\left(n-\frac{1}{2}\right) \pi i}$ gegen seinen Werth in $\int_{\infty i}^{+1}$ angenommen hat, so hat man:

$$
\int_{\infty i}^{+1}+\int_{+1}^{\infty i}=\left(e^{4\left(n-\frac{1}{2}\right) \pi i}-1\right) \int_{1}^{\infty i}=2 i e^{2 n \pi i} \sin 2 n \pi \int_{1}^{\infty i},
$$

wo letzteres Integral auf der linken Seite des Schnittes zu nehmen ist. Für ein ganzes $n$ verschwindet in:

$$
\int_{\infty i}^{\infty i}=\int_{+1}^{+1}+2 i e^{2 n \pi i} \sin 2 n \pi \int_{1}^{\infty i}
$$

des Factors sin $2 n \pi$ wegen, das letate Integral; dagegen bleibt in jedem Falle:

$$
2 i e^{8 n \pi i} \int_{1}^{\infty i}=\frac{\int_{\infty i}^{\infty i}-f_{1}^{+1}}{\sin 2 n \pi}
$$

von Null verschieden and giebt eine Lösung unserer Differentialgleichung, wenn sie mit $x^{n}$ multiplicirt wird, und zwar findet man vermittels der Formeln (4) und $\left(6^{a}\right)$ des vorigen \&. für $J^{n}(x)$ und $J^{-n}(x)$ die Gleichung:

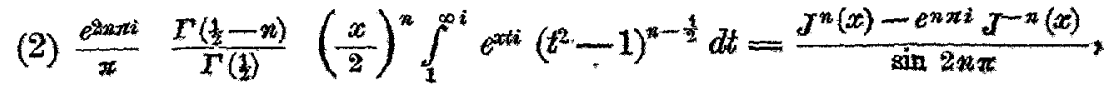


wo die rechte Seite nach (2) in \$. 1.

$$
=\frac{1}{2 \pi} Y^{n}(x)-\frac{i}{2} \frac{e^{n \pi i}}{\cos n \pi} J^{n}(x)
$$

ist, und bleibt, wenn $n$ auch im Speciellen eine ganze Zahl ist*).

Man bemerkt, dass

$$
\int_{-1}^{\infty i}=\int_{-1}^{+1}+\int_{+1}^{+\infty i}
$$

wenn man linker Hand von dem Schnitte integrirt, gesetzt werden kann, weil zwischen den Wegen dieser Integrale die Function überall stetig and endlich bleibt, und es jst hieraus leicht die Gleichung:

$$
\frac{e^{2 n \pi i}}{\pi} \frac{\Gamma\left(\frac{1}{2}-n\right)}{\Gamma\left(\frac{1}{2}\right)}\left(\frac{x}{2}\right)^{n} \int_{-1}^{\infty} e^{x \pi i}\left(t^{2}-1\right)^{n-\frac{1}{2}} d t=e^{n \pi i} \frac{e^{n \pi i} J^{n}(x)-J^{-n}(x)}{\sin 2 n \pi}
$$
abzuleiten, wo die rechte Seite nach (3) in $\S .1$.

$$
=\frac{1}{2 \pi} Y^{n}(x)+\frac{i}{2} \frac{e^{n \pi i}}{\cos n \pi} J^{n}(x)
$$

für jedes $n$ gesetzt werden kann.

Es sind nun in den Formeln (1) des vorigen \$. und (2) ses §. unter der Voraussetzung eines $n$ mit positivem reellen Theile die Integrale von einem Verzweigungspunkte zum andern, durch die $J$ und $Y$ dargestellt. Auch sind bereits in (4) und $\left(6^{a}\right)$ des vorigen $\$$. Integrale behandelt worden, welche ron einem Verzweigungspunkte ausgehend, wieder zu iḥm zurückkehren. Solcher Integrale aber haben wir noch zwei zu entwickeln:

Die bisher immer angewandte Zerschneidung von -1 bis +1 und dann parallel der positiven imaginären Axe hinaus in's Unendliche, ist, wie schon früher bemerkt, nur eine arbiträre, da es sich im Wesentlichen bei der Zerschneidung darum handelt, ein Umlaufen eines Verzweigungspunktes $-1,+1, \infty$ unmöglich zu machen. Wir können daher auch die in Fig. 4. gewählte Zerschneidung von +1 nach $1+\infty i$, und ron -1 in's Unendliehe in ganz beliebiger Richtung zu Grunde legen. Bestimmen wir dann den Werth der Potenz linkerseits der Linien von 1 bis $1+\infty i$ ganz wie früher, so behält das auf der linken Seite gewonnene Integral:

$$
\int_{1}^{\infty i} e^{x t i}\left(t^{2}-1\right)^{n-\frac{1}{2}} d t
$$

*) Setzt man in (2), $t=\cos (\varphi i)$, so entspricht dem $t=1$ die untere Grenze $\varphi=0$; für $\varphi=\infty+\frac{\pi}{2} i$ aber wird $\cos \varphi i=\infty i$ und es verwandelt sich daber jenes Integral in das von Hrn. Heine a. a. O. angegebene Integral:

$$
\int_{0}^{\infty+\frac{\pi}{2} i} e^{x i \cos (\varphi p i)} \sin ^{2 n}(\varphi i) d \varphi \text {. }
$$


seinen in (2) gefundenen Werth. Betrachten wir nun $\int_{\infty i}^{\infty i}$ derselben. Function von $\infty i$ links von dem Schnitte um $t=+1$ herum und auf der rechten Seite des Schnittes wieder in das unendlich Imaginäre hinaus, wie es etwa in Fig. 4. gezejchnet ist, so stellt dies eine nach $n$ immer stetige Function dar, deren Werth wir ermitteln können für ein $n$ mit positivem reellem Theile; denn in diesem Falle lässt sich das Integral dicht an den Schnitt zusammenziehen und man firdet, da $\left(t^{2}-1\right)^{n-\frac{1}{2}}$ rechts von dem Schnitte den Factor $e^{2\left(n-\frac{1}{3}\right) \pi i}$ gegen seinen Werth auf der linken Seite angenommen hat:

$$
\int_{\infty i}^{\infty}=-2 \cos n \pi e^{n \pi i} \int_{1}^{\infty i},
$$

Fig. 4 .

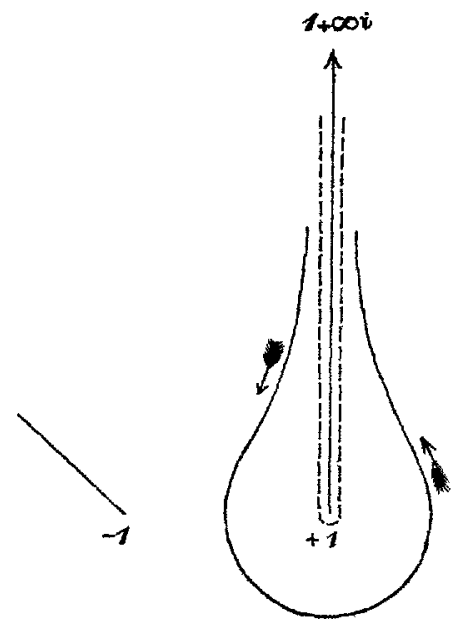

wo das letzte Integral links vom Schnitte zu nehmen ist, so dass man nach (2) die Gleichung:

$\left(6^{b}\right)-\frac{1}{2 \pi} \frac{e^{n \pi i}}{\cos n \pi} \frac{\Gamma\left(\frac{1}{2}-n\right)}{\Gamma\left(\frac{1}{2}\right)}\left(\frac{x}{2}\right)_{\infty i}^{n} \int_{\infty i}^{\infty} e^{x t i}\left(t^{2}-1\right)^{n-\frac{1}{2}} d t=\frac{J^{n}(x)-e^{n \pi i} J^{-n}(x)}{\sin 2 n \pi}$

erhält, die nun für jedes $n$, auch für solche mit negativem reellen Theile gilt.

Wenn wir die Zerschneidung von -1 in's Unendliche in einer Linie von -1 parallel der positiven imaginären Axe vornehmen, wie in Fig. 5., und bestimmen $\left(t^{2}-1\right)^{n-\frac{1}{2}}$ rechterseits dieses Schnittes so, wie vorhin, so reducirt sich das $\int_{\infty i}^{\infty i}$ in positiver Richtung um $t=-1$ herumgenommen, für ein $n$ mit positivem reellen Theile auf:

$$
\int_{\infty i}^{\infty i}=+2 \cos n \pi e^{-n \pi i} \int_{-1}^{\infty i},
$$

Fig. 5.

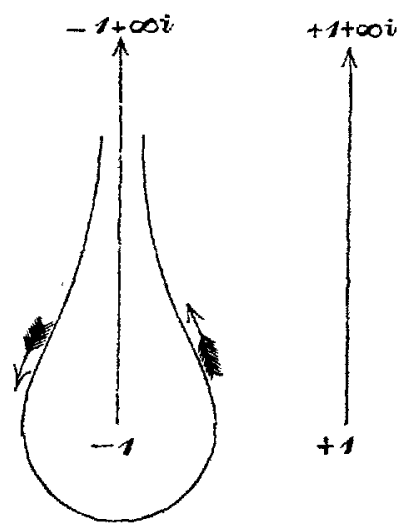

wo letzteres Integral in demselben Sinne, als in (3) $\mathrm{zu}$ nehmen ist, so dass man jetzt die wiederum für alle $n$ gültige Formel:

$\left(6^{c}\right)+\frac{1}{2 \pi} \frac{e^{n \pi i}}{\cos n \pi} \frac{\Gamma\left(\frac{1}{2}-n\right)}{\Gamma\left(\frac{1}{2}\right)}\left(\frac{x}{2}\right)^{n} \int_{\infty i}^{\infty i} e^{x t i}\left(t^{2}-1\right)^{n-1} d t=e^{n \pi i e^{n \pi i} J^{n}(x)-J^{-n}(x)}$, erhalten wird. - 
Es sind somit schliesslich alle in \$. 2. aufgestellten Integrale der Differentialgleichung in \$\$. 3. und 4. (wo sie gleichbeziffert erscheinen) durch die $J$ und $Y$ dargestellt, und das ganze System der möglichen Integrale erschöpft.

\section{§. 5 .}

\section{Die Cylinderfunctionen zweiter Art.}

Für den Fall eines positiven ganzen n, dem wir diesen Paragraphen widmen, haben wir in den Formeln (2), (3) des vorigen Paragraphen

$\frac{(-1)^{n}}{\Gamma\left(n+\frac{1}{2}\right) \Gamma\left(\frac{1}{2}\right)}\left(\frac{x}{2}\right)^{n} \int_{1}^{\infty i} e^{x t i}\left(t^{2}-1\right)^{n-\frac{1}{2}} d t=\frac{1}{2 \pi} Y^{n}(x)-{ }_{2}^{i} J^{n}(x)$

(3) $\frac{(-1)^{n}}{\Gamma\left(n+\frac{1}{2}\right) \Gamma\left(\frac{1}{2}\right)}\left(\frac{x}{2}\right)^{n} \int_{-1}^{\infty i} e^{x t i}\left(t^{2}-1\right)^{n-\frac{1}{2}} d t=\frac{1}{2 \pi} Y^{n}(x)+\frac{i}{2} J^{n}(x)$

zwei Formeln gewonnen, durch deren Addition man $Y$ allein darstellen kann.

Doch können diese Formeln noch in eigenthümlicher Weise transformirt werden: In den vorliegenden Integralen sind nur $t= \pm 1$ die Verzweigungspunkte der Function unter dem Integralzeichen und der Schnitt von 1 bis $\infty i$ ist überflüssig; führen wir aber gleichzeitig $\log (-t)$ ein, so stellt sich wieder ein von $t=0$ ins Unendliche lanfender Schnitt als nothwendig dar, um $\log (-t)$ in der Ebene eindeutig bestimmen zu können. Demnach behalten wir den Schnitt von -1 über +1 nach $1+\infty i$ bei, bestimmen $\left(t^{2}-1\right)^{x-\frac{1}{2}}$ zu beiden Seiten des Schnittes von -1 bis +1 nach den Formeln (I), (II) des \$. 3. und setzen

$$
t=r e^{\varphi i}, \log (-t)=\log r+(\varphi-\pi) i,
$$

so dass also $\log (-t)$ für ein negatives reelles $t$ reell ist (log $r$ immer reell genommen).

Wir bilden nun unter der Voraussetzung eines ganzen $n$ das

$$
\int_{\infty i}^{\infty i} e^{x t i}\left(t^{2}-1\right)^{n-\frac{1}{2}} \log (-t) d t
$$

von der linken Seite des Schnittes um \pm 1 herum nach der rechten Seite desselben (Fig. 2.); ziehen die Integrationscurve dicht an den Schnitt zosammen, und zerlegen das Integral:

$$
\int_{\infty i}^{\infty i}=\int_{\infty i}^{1}+\int_{+1}^{+1}+\int_{1}^{\infty i}
$$


Es wird nun die Potenz in $\int_{\infty i}^{1}$ linkerseits am Schnitte dieselben Werthe haben, als in $\int_{1}^{\infty i}$ rechterseits; dagegen bat $\log (-t)$, weil unterdessen der Punkt $t=0$ nmkreist ist, in letzterem $\int_{1}^{\infty i}$ um $2 \pi i$ zugenommen, und man hat somit:

also:

$$
\int_{\infty}^{1}+\int_{i}^{\infty i}=2 \pi i \int_{1}^{\infty i} e^{x t i}\left(t^{2}-1\right)^{n-\frac{1}{2}} d t
$$

$$
2 \pi i \int_{1}^{\infty i} e^{x t i}\left(t^{2}-1\right)^{n-\frac{1}{2}} d t=\left(\int_{\infty i}^{\infty i}-\int_{1}^{1}\right) e^{z t i}\left(t^{2}-1\right)^{n-\frac{1}{2}} \log (-t) d t
$$

so dass man aus (2), wenn man den Werth von $J^{-n}(x)=(-1)^{n} J^{n}(x)$ aus $\left(6^{c}\right)$ in $\$ .3$ substituirt:

erhält, wo:

$$
Y^{n}(x)=L^{n}(x)+E^{n}(x)
$$

$$
\begin{gathered}
L^{n}(x)=-i \frac{(-1)^{n}}{\Gamma\left(n+\frac{1}{2}\right) \Gamma\left(\frac{1}{2}\right)}\left(\frac{x}{2}\right)^{n} \int_{\infty i}^{\infty i} e^{x t i}\left(t^{2}-1\right)^{n-\frac{1}{2}}\left[\log (-t)-\frac{\pi}{2} i\right] d t \\
E^{n}(x)=+i \frac{(-1)^{n}}{\Gamma\left(n+\frac{1}{2}\right) \Gamma\left(\frac{1}{2}\right)}\left(\frac{x}{2}\right)^{n *} \int_{i}^{1} e^{x t i}\left(t^{2}-1\right)^{n-\frac{1}{2}} \log (-t) d t .
\end{gathered}
$$

Letateres Integral kann noch einer weiteren Reduction unterzogen werden. Bemerkt man nämlich, dass $\left(t^{2}-1\right)^{n-\frac{1}{2}}$ zu beiden Seiten der Strecke von 0 bis +1 entgegengesetzte Werthe hat, $\log (-t)$ aber unterhalb derselben um $2 \pi i$ grösser ist, als oberhalb, so findet man die beiden Theile von $\int_{+1}^{+1}$ :

$$
\int_{+1}^{0}+\int_{0}^{+1}=-2 \int_{0}^{1} e^{x t i}\left(t^{2}-1\right)^{n-\frac{1}{2}} \log t d t
$$

wo dies letzte Integral oberhalb des Schnittes genommen werden muss und $\log (-t)+\pi i=\log t$ gesetzt, also $\log t$ innerhalb des Integrationsintervalles reell ist.

Was ferner die anderen Theile des Intègrales $\vec{f}_{0}^{-1}, \int_{-1}^{0}$ betrifft, so hat die Potenz in dem zweiten Integrale ebenfalls das entgegengesetzte Zeichen, $\log (-t)$ aber denselben Werth und es ist:

$$
\int_{0}^{-1}+\int_{-1}^{0}=2 \int_{0}^{-1} e^{x t i}\left(t^{2}-1\right)^{n-\frac{1}{2}} \log (-t) \cdot d t
$$

oder nach Vertauschung von $t$ mit $-t$ :

$$
=-2 \int_{0}^{1} e^{-\alpha t i}\left(t^{2}-1\right)^{n-\frac{1}{2}} \log t d t,
$$


wo dies Integral ebenfalls oberhalb des Schnittes zu nehmen ist. Śomit hat man, wenn man noch $\left(t^{2}-1\right)^{n-\frac{1}{2}}=(-1)^{n} i\left(1-t^{2}\right)^{n-\frac{1}{2}}$ nach (I) in \$. 3. setzt:*)

$$
E^{*}(x)=\frac{4}{\Gamma\left(n+\frac{1}{2}\right) \Gamma\left(\frac{1}{2}\right)}\left(\frac{x}{2}\right)^{n} \int_{0}^{1} \cos x t \cdot\left(1-t^{2}\right)^{n-\frac{1}{2}} \log t d t .
$$

Von Herm Neumann ist ein zu dieser Art gehöriges Integral für $Y^{0}(x)$ gegeben worden, welches, wie mir scheint, eben nur für $n=0$ in dieser Weise existirt. Ich erhalte dasselbe einfach als Grenzfall, indem ich neben (1) in \$. 3, noch das andere Integral:

$$
J^{-n}(x)=\frac{1}{\Gamma\left(\frac{1}{2}-n\right) \Gamma\left(\frac{1}{2}\right)}\left(\frac{x}{2}\right)^{-n} \int_{-1}^{+1} e^{z t i}\left(1-t^{2}\right)^{-n-\frac{3}{2}} d t
$$

setze, welches unter der Voraussetzung eines der Null nahen reellen Theiles von $n$ gleichzeitig mit jenem $J^{n}(x)$ besteht. Bildet man dann:

$$
\begin{gathered}
Y^{n}(x)=2 \pi e^{x \pi i} \frac{J^{*}(x) \cos n \pi-J^{-n}(x)}{\sin 2 n \pi} \\
=\frac{2 \sqrt{\pi} e^{n \pi i}}{\sin 2 n \pi} \int_{-1}^{+1} e^{x t i} \frac{d t}{\sqrt{1-t^{*}}}\left\{\frac{\left(\frac{x}{2}\right)^{n}\left(1-t^{2}\right)^{n}}{\Gamma\left(\frac{1}{2}+n\right)} \cos n \pi-\frac{\left(\frac{x}{2}\right)^{n}\left(1-t^{2}\right)^{-n}}{\Gamma\left(\frac{1}{2}-n\right)}\right\},
\end{gathered}
$$

und lässt $n$ zur Grenze Null übergehen, so findet man durch Differentiation der letzten Klammergrösse und des verschwindenden Nenners $\sin 2 n \pi$ :

welches mit

$$
Y(x)=\frac{2}{\pi} \int_{-1}^{+1} e^{x t i} \frac{d t}{\sqrt{1-t^{2}}}\left\{\log \left(1-t^{2}\right)-\psi\left(\frac{1}{2}\right)\right\}
$$

$$
J(x)=\frac{1}{x} \int_{-1}^{+1} e^{x t i} \frac{d t}{V I-t^{2}}
$$

zosammen, für $t=\cos \varphi$ in die erwähute Formel**) übergeht. -

Die obigen Integrale $L$ und $E$ gestatten eine directe Kntwickelung in die Reihe, welche in §. 1. für die $Y$ function gegeben ist. Was zunäehst $E$ betrifft, so entwickele man $\cos x t$ in eine Reihe und bediene sich dann zur Integration der aus:

$$
\int_{0}^{1} t^{2 p}\left(1-t^{2}\right)^{n-\frac{1}{2}} d t=\frac{1}{2} \frac{\Gamma\left(p+\frac{1}{2}\right) \Gamma\left(n+\frac{1}{2}\right)}{\Gamma(p+n+1)}
$$

*) Wenn man hierin $t=\cos \varphi$ setet, so erhält man ein Integral, welches dem von Herrn Lommel (s. p. 468) gegebenen ähalich, aber nicht gleich ist.

**) Theorie d. Bess. Fonct. p. 46 Formel (27) und (28). Man beachte übrigens, dass schon oben p. 472 anf den Unterschied meiner und der Newmann'schen Bezeichnung aufmerksam gemacht wurde. 
durch Differentiation nach $p$ folgenden Formel:*)

$\int_{0}^{1} t^{2 p}\left(1-t^{2}\right)^{n-\frac{1}{2}} \log t d t=\frac{1}{4} \frac{\Gamma\left(p+\frac{1}{2}\right) \Gamma\left(n+\frac{1}{2}\right)}{\Gamma(p+n+1)}\left\{\psi\left(p+\frac{1}{2}\right)-\psi(p+n+1\}\right.$.

Dann findet man:

$E^{n}(x)=\left(\frac{x}{2}\right)^{n} \sum_{p=0}^{\infty} \frac{(-1)^{p}}{\Gamma(p+1) \Gamma(p+n+1)}\left(\frac{x}{2}\right)^{2 p}\left\{\psi\left(p+\frac{1}{2}\right)-\psi(p+n+1)\right\}$

Was nun die Entwickelung von $L^{n}(x)$ betrifft, so geschieht sie ähnlich, wie die des verwandten Integrales p. 480-482. Man findet zunächst:

$L^{n}(x)=(-1)^{n} \frac{i}{\sqrt{\pi}}\left(\frac{x}{2}\right)^{n} \sum_{p=0}^{\infty} \frac{(-1)^{p}}{\Gamma\left(n-p+\frac{1}{2}\right) \Gamma(p+1)} \int_{\infty i}^{\infty i} e^{x t i} t^{2 n-2 p-1}\left[\log (-t)-\frac{\pi}{2} i\right] d t$.

Um den Werth der Integrale, oder allgemeiner:

$$
\int_{\infty i}^{\infty i} e^{x t i}(-t)^{q}\left[\log (-t)-\frac{\pi}{2} i\right] d t
$$

für jedes reelle zu $q$ ermitteln, setzen wir, wie p. 481, $t=u i$. Wenn nun hier $(-t)^{q}$ so bestimmt wird, dass es für reelle negative $t$ reell wird, und $(-u)^{q}$ in derselben Weise, so ist $u=r e^{\varphi i},(-u)^{q}=r^{q} e^{q(s-\pi) i}$ und daher für $u=i=e^{\frac{\pi}{2} i},(-u)^{q}=e^{-q \frac{\pi}{2} i}$. Um nun in $(-t)^{q}=(-u)^{q} i^{q}$ die Potenz von $i^{q}$ zu bestimmen, setzen wir $t=-1, u=i$ und finden so:

$$
(-t)^{q}=e^{q \frac{\pi}{2} i}(-u)^{q} .
$$

Wird num $\log (-t)$ ebenfalls für negative reelle $t$ reell bestimmt und $\log (-u)$ auch so, so ist $u=i$ gesetzt $\log (-u)=-\frac{\pi}{2} i$ und daher:

$$
\log (-t)=\log (-u)+\frac{\pi}{2} i
$$

*) Ich wähle hier zur Entwickelung die Form von $E^{n}(x) \operatorname{durch} \int_{0}^{1}$, anstatt der obigen durch $\int_{1}^{I}$, um alle Wertlangigkeiten zu vermeiden, welche durch die Verallgemeinerung des Euler'schen Integrales erster Gattung entspringen würden. Ich habe übrigens bereits a. a. 0 . in Schlömilch's Journ. t. IX. p. 13 diese allgemeinste Formel in der Gestalt:

$$
\begin{gathered}
\int_{b}^{b}(t-a)^{p}(t-b)^{q}(t-c)^{-p-q-2} d t \\
=2 \pi i(b-c)^{-p-1}(c-a)^{-q-1}(a-b)^{p+q+1} \frac{\Gamma(q+1)}{\Gamma(-p) \Gamma(p+q+2)}
\end{gathered}
$$

gegeben, wo von $b \mathrm{um}$ den Verzweigangspunkt $a$ herum nach $b$ zurück zu integriren ist; $a, b, c, q$ beliebige complexe Grössen sind und nur $q$ der Bedingung unterhiegt, dass sein reeller Theil grǒsser als -1 ist. 
Man erhält so unter denselben Voraussetzungen in Bezug auf die Zerschneidung in der $u$ Ebene als p. 481 :

$$
\int_{\infty}^{\infty i} e^{x t i}(-t)^{q}\left[\log (-t)-\frac{\pi}{2} i\right] d t=i e^{q} \frac{\pi}{2} i \int_{\infty}^{\infty} e^{-x u}(-u)^{q} \log (-u) d u .
$$

Letztere Integrale können nun aber aus der dort p. 482 ebenfalls erwiesenen Gleichung:

$$
\int_{\infty}^{\infty} e^{-u x}(-u)^{q} d u=-\frac{2 \pi i}{x^{q+1}} \frac{1}{\Gamma(-q)}
$$

leicht durch Differentiation nach $q$ abgeleitet werden, die

$$
\int_{\infty}^{\infty} e^{-u x}(-u)^{q} \log (-u) d u=\frac{2 \pi i}{x^{q+1} \Gamma(-q)}\{\log x-\psi(-q)\}
$$

ergiebt, and zwar unbedingt für jedes $q$. Indessen bemerke man, dass, wenn $q$ eine positive ganze Zahl ist, $\Gamma(-q)$ und $\psi(-q)$ gleichzeitig unendlich werden, und zwar, wie man bei Anwendung der Formel $\Gamma(-q) \Gamma(1+q) \sin q \pi=-\pi$ leicht findet, so, dass

$$
\frac{\psi(-q)}{\Gamma(-q)}=(-1)^{q+1} \Gamma(q+1) \text {. }
$$

Damit geht vorstehende Formel für ein solches $q$ über in die andere:

$$
\int_{\infty}^{\infty} e^{-u x}(-u)^{q} \log (-u) d u=(-1)^{q} \frac{2 \pi i}{x^{q+1}} \Gamma(q+1),
$$

die man auch leicht direct findet, wenn man beachtet, dass für ein ganzes positives $q,(-v)^{q}$ keinen Verzweigungspunkt in $u=0$ hat, der Integrationsweg also auf die reelle Abscissenaxe zusammengezogen werden kann.

Wendet man num diese Formel auf obige Summe von $p=0$ bis $p=n-1$ an; die erste aber von $p=n$ an und führt in der letzten Summe einen neuen Summationsbuchstaben $p$ für $p-n$ ein, so dass die Summe wieder bei $p=0$ beginnt, so findet man:

$$
\begin{gathered}
L^{n}(x)=-\left(\frac{x}{2}\right)^{-n} \sum_{p=0}^{n-1} \frac{\Gamma(n-p)}{\Gamma(p+1)}\left(\frac{x}{2}\right)^{2 p} \\
+\sum_{p=0}^{\infty} \frac{(-1)^{p}}{\Gamma(p+1) \Gamma(p+n+1)}\left(\frac{x}{2}\right)^{2 p}\left\{\log x^{2}-2 \psi(2 p+1)\right\} .
\end{gathered}
$$

Benutzt man dann die ans:

hervorgehende Relation:

$$
\Gamma\left(\frac{1}{2}\right) \Gamma(2 p+1)=2^{2 p} \Gamma(p+1) \Gamma\left(p+\frac{1}{2}\right)
$$

$$
2 \psi(2 p+1)=2 \log 2+\psi(p+1)+\psi\left(p+\frac{1}{2}\right)
$$


so erhält man durch Addition

$$
Y^{n}(x)=L^{n}(x)+E^{n}(x)
$$

vollkommen in Uebereinstimmung mit der \$. 1. entwickelter Reihe.

§. 6 .

\section{Die semiconvergenten Entwickelungen der Cylinderfunetionen.}

Um semiconvergente Reihen für die Cylinderfunctionen, zunächst unter der Voraussetzung, dass $x$ und $n$ reelle positive Theile haben, zu entwickeln, gehen wir aus von dem

$$
\int_{1}^{\infty i} e^{x t i}\left(t^{2}-1\right)^{n-\frac{1}{2}} d t
$$

welches im Sinne von (2) des §. 4. auf der linken Seite des Schnittes von 1 bis $1+\infty i$, geradlinig zu erstrecken ist.

Wir transformiren dies Integral, indem wir $t=1+r i$ setzen und hier $r$ im Reellen von 0 bis $\infty$ wachsen lassen. Dann ist $t-1=r e^{\frac{\pi}{2} i}$ und nach den Festsetzungen in \$. 3 .

$$
(t-1)^{n-\frac{1}{2}}=r^{n-\frac{1}{2}} e^{\left(n-\frac{1}{2}\right) \frac{\pi}{2} i} .
$$

Ferner ist $t+1=2\left(1+\frac{r i}{2}\right)$ also:

$$
(t+1)^{n-\frac{1}{2}}=2^{n-\frac{1}{2}} e^{-2\left(n-\frac{1}{2}\right) \pi i}\left(1+\frac{r i}{2}\right)^{n-\frac{1}{2}} .
$$

wo $\left(1+\frac{r i}{2}\right)^{n-\frac{1}{2}}$ so zu nehmen ist, dass es für $r=0$ sich anf +1 reducirt. Denn dann wird eben für $\gamma=0,(t+1)^{n-\frac{1}{2}}=2^{n-\frac{1}{2}} e^{-\cdot 2\left(n-\frac{1}{2}\right) \pi i}$, welches ebenfalls mit den Festsetzungen in $\$$. 3. über die Zerschneidung in Bezug auf $(t+1)^{q}$ übereinstimmt. Somit gibt diese Substitution in (2) des $\$$. 4.:

$\frac{{ }^{n}(x)-e^{n \pi i} J^{-n}(x)}{\sin 2 n \pi}=\frac{-i}{\pi \sqrt{2}} \frac{\Gamma\left(\frac{1}{2}-n\right)}{\Gamma\left(\frac{1}{2}\right)} x^{n} e^{x i+\frac{1}{2}\left(n-\frac{1}{2}\right) n i} \int_{0}^{\infty} e^{-x \pi} r^{n-\frac{1}{2}}\left(1+\frac{r i}{2}\right)^{n-\frac{i}{2}} d r$.

Ebenso gibt die Substitution von $t=-1+r i$ in (3) des §. 4. nach Bestimmung der richtigen Zeichen:

$$
\frac{n \pi i}{e^{n \pi i} J^{n}(x)-J^{-n}(x)}=\frac{+i}{\pi \sqrt{2}} \frac{\Gamma\left(\frac{1}{2}-n\right)}{\Gamma\left(\frac{1}{2}\right)} x^{n} e^{-x i+1\left(3 n+\frac{1}{2}\right) \pi i} \int_{0}^{\infty} e^{-x r} \gamma^{m-\frac{1}{2}}\left(1-\frac{r i}{2}\right)^{n-\frac{1}{2}} d r \text {. }
$$

Um nun diese Integrale nach absteigenden Potenzen von $x$ zu entwickeln, müssen

$$
\left(1 \pm \frac{r i}{2}\right)^{n-\frac{1}{2}}
$$

nach aufsteigenden Potenzen von $r$ entwiekelt werden; in convergenter 
Weise ist dies nur für den Theil des Integrationsintervalles möglich, in dem $r<2$ ist; in dem Intervall von $r=2$ bis $r=\infty$ aber wird die Reihe eine semiconvergente werden.

Erinnern wir uns der Lagrange'schen Form des Restes in der Taylor'schen Entwickelung, wonach, wenn $f(r)$ eine reelle Function von $r$ bedeutet:

$$
f(r)=f(0)+\frac{r}{1} f^{\prime}(r)+\ldots+\frac{r^{m-1}}{\Gamma(m)} f^{m-1}(0)+\frac{r^{m}}{\Gamma(m+1)} f^{m}(h)
$$

und $h$ eine gewisse reelle Grösse in dem Intervalle zwischen 0 und $r$ darstellt, so haben wir, wenn wir den Satz auf die reelle Grösse

$$
f(r, n)=\left(1+\frac{r i}{2}\right)^{n-\frac{1}{2}}+\left(1-\frac{r i}{2}\right)^{n-\frac{1}{2}}
$$

anwenden und einstweilen

$$
n_{p}=\frac{\Gamma\left(n+\frac{1}{2}\right)}{\Gamma\left(n-p+\frac{1}{2}\right) \Gamma(p+1)}
$$

setzen, die Entwickelung:

$$
\dot{f}(r, n)=2 \sum_{p=0}^{m-1} n_{2 p}\left(\frac{r i}{2}\right)^{2 p}+n_{2 m}\left(\frac{r i}{2}\right)^{2 m}\left\{\left(1+\frac{h i}{2}\right)^{n-\frac{1}{2}-2 m}+\left(1-\frac{h i}{2}\right)^{n-\frac{1}{2}-2 m}\right\}
$$

Nimmt man nun $2 m>n-\frac{1}{2}$ an, so ist der Modul

$$
\bmod \left(1 \pm \frac{h i}{2}\right)^{n-\frac{1}{2}-2 m}=\left(1+\frac{h^{2}}{4}\right)^{\frac{1}{2}\left(n-\frac{1}{2}-2 m\right)}<1
$$

und daher der Modul der Summe dieser Binome kleiner als 2; so dass der hinzugefügte Rest jedenfalls numerisch kleiner ist, als das an seine Stelle tretende Glied der regulär fortgesetzten binomischen Reihe.

Die Gültigkeit dieser Schlüsse beruht auf der stillschweigend gemachten Voraussetzung eines reellen $n$; ist aber $n$ complex, und bezeichnet man mit $n^{\prime}$ die conjugirte imaginäre Grösse, so entwickele man die jedenfalls reelle Grösse

in derselben Weise uud erhält so:

$$
f(r, n)+f\left(r, n^{\prime}\right)
$$

$$
\begin{gathered}
f(r, n)+f\left(r, n^{\prime}\right)=2 \sum_{p=0}^{m-1}\left(n_{2 p}+n_{2 p}^{\prime}\right)\left(\frac{r i}{2}\right)^{2 p} \\
+n_{2 m}\left(\frac{r i}{2}\right)^{2 m}\left\{\left(1+\frac{h i}{2}\right)^{n-\frac{1}{2}-2 m}+\left(1-\frac{h i}{2}\right)^{n-\frac{1}{2}-2 m}\right\} \\
+n_{2 m}^{\prime}\left(\frac{r i}{2}\right)^{2 m}\left\{\left(1+\frac{h i}{2}\right)^{n^{\prime}-\frac{1}{2}-2 m}+\left(1-\frac{h i}{2}\right)^{n^{\prime}-\frac{1}{2}-2 m}\right\} .
\end{gathered}
$$

Das entsprechende Glied der binomischen Reihe, wenn sie regulär fortgesetzt wird, ist:

$$
2 n_{2 m}\left(\frac{\pi i}{z}\right)^{2 n}+2 n_{2 m}^{n}\left(\frac{r i}{2}\right)^{2 m}
$$


und jener Rest geht aus diesem hervor, wenn die zwei Glieder mit complexen Grössen multiplicirt werden, deren Modulus kleiner als 1 ist, sobald $2 m$ den reellen Theil von $\left(n-\frac{1}{2}\right)$ übersteigt; der Rest ist also kleiner als dies Glied.

Ganz ebensolehe Betrachtungen würde man über die Reihe für die reelle Grösse

$$
\frac{1}{i}\left[f(r, n)-f\left(r, n^{\prime}\right)\right]
$$

anstellen können, und sieht nun, dass, wenn man letztere mit $i$ multiplicirt und zar ersteren addirt, eine Reihe:

$$
f(r, n)=\left(1+\frac{r i}{2}\right)^{n-\frac{1}{2}}+\left(1-\frac{r i}{2}\right)^{n-\frac{1}{2}}=2 \sum n_{2 p}\left(\frac{r i}{2}\right)^{2 p}
$$

entsteht, welche die Eigenschaft hat, dass der Rest, den man rechter Hand hinzufügen müsste, wenn man die Reihe an irgend einer Stelle abbricht, sowohl in seinem reellen als imaginären Theile kleiner ist, als das regulär folgende Glied. Jedoch gilt diese Eigenschaft, welche man als Semiconvergenz bezeichnet, erwiesener Massen erst von dem Gliede an, wo $2 p$ grösser als der reelle Theil von $\left(n-\frac{1}{2}\right)$ ist.

Es ist nun einleuchtend, dass man diese Betrachtung ohne Weiteres auf die Entwickelung des reellen Ausdruckes:

$$
\frac{1}{i}\left\{\left(1+\frac{r i}{2}\right)^{n-\frac{1}{2}}-\left(1-\frac{r i}{2}\right)^{n-\frac{1}{2}}\right\}=\frac{2}{i} \sum n_{2 p+1}\left(\frac{r i}{2}\right)^{2 p+1}
$$

übertragen kann und man erhält so, wenn man diese Reihe mit $i$ multiplicirt und zur vorigen addirt oder subtrahirt:

$$
\left(1 \pm \frac{r i}{2}\right)^{n-\frac{1}{2}}=\Sigma n_{2 p}\left(\frac{r i}{2}\right)^{2 p} \pm \Sigma n_{2 p+1}\left(\frac{r i}{2}\right)^{2 p+\lambda}
$$

wo beide Reihen von der Art sind, dass, wenn man bei einem Gliede derselben die numerische Berechnung abbricht, die so erhaltene complexe Grösse von dem Werthe, welchen die Reihe streng darstellen soll, ım eine Grösse differirt, welche in ihrem reellen, sowie ihrem imaginären Theile numeriseh unter den entsprechenden Theilen des in der Reihe nächstfolgenden Gliedes liegt.

Will man der Einfachheit wegen beide Reihen in die eine:

$$
\left(1 \pm \frac{r i}{2}\right)^{n-\frac{1}{2}}=\Sigma n_{p}\left( \pm \frac{r i}{2}\right)^{p}
$$

vereinigen, so darf man doch nie vergéssen, dass die Glieder gerader Ordnung ebenso wie die nngerader eigentlich eine Reihe für sich bilden.

Wenn man vorstehende-Reihe mit einer complexen Grösse multiplicirt, so wird man nicht in allen Fällen behaupten können, dass der Rest in seinen beiden Theilen jederzeit unter den entsprechenden Theilen des nächsten Gliedes liegen müsse. Die Figenthtimlichkeit 
unserer semiconvergenten binomischen Reihe kann aber auch so ausgesprochen werden, dass der Modul (der absolute Betrag, wie ihn Herr Weierstrass treffend nennt) des Restes unter dem des nächsten Gliedes liegen müsse; und diese Eigenschaft wird bei jeder Multiplication mit einer complexen Grösse erhalten. Ebenso wenig wird dieselbe durch eine Integration der Reihe anfgehoben.

Substituiren wir nun diese Reihen in obigen Integralen, so findet man ohne Schwierigkeit:

(1) $\frac{J^{n}(x)-e^{n \pi i} J^{-n}(x)}{\sin 2 n \pi}=-\frac{i}{\sqrt{2 \pi x}} \frac{e^{n \pi i}}{\cos n \pi} \sum \frac{(n \cdot p)}{(2 x)^{p}} e^{\left[x-\frac{1}{2} \pi\left(n+\frac{1}{2}-p\right)\right] i}$

(2) $e^{n \pi i} \frac{e^{n \pi i} J^{n}(x)-J^{-n}(x)}{\sin 2 n \pi}=+\frac{i}{\sqrt{2 \pi x}} \frac{e^{n \pi i}}{\cos n \pi} \Sigma \frac{(n, p)}{(2 x)^{p}} e^{-\left[x-\frac{1}{2} \pi\left(n+\frac{1}{2}-p\right)\right] i}$

wo zur Abkürzung

$$
(n, p)=\frac{\Gamma\left(n+p+\frac{1}{2}\right)}{\Gamma\left(n-p+\frac{1}{2}\right)} \frac{1}{\Gamma(p+1)}=\frac{n^{2}-\left(\frac{1}{2}\right)^{2}}{1} \cdot \frac{n^{2}-\left(\frac{3}{2}\right)^{2}}{2} \cdots \frac{n^{2}-\left(\frac{2 p-1}{2}\right)^{2}}{p}
$$

gesetzt ist.

Beachtet man, dass nach (2) und (3) in \$. 1. die linken Seiten von (1), (2) respective

$$
\frac{1}{2 \pi} Y^{n}(x) \mp \frac{i}{2} \frac{e^{n \pi i}}{\cos n \pi} J^{n}(x)
$$

sind, so erhält man durch Subtraction und Addition:

$$
J^{n}(x)=\sqrt{\frac{2}{\pi x}} \sum \frac{(n, p)}{(2 x)^{p}} \cos \left[x-\frac{1}{2} \pi\left(n+\frac{1}{2}-p\right)\right] .
$$

(4) $e^{-k \pi i} \cos n \pi \frac{1}{\pi} Y^{n}(x)=\sqrt{\frac{2}{\pi x}} \Sigma \frac{(n, p)}{(2 x)^{p}} \sin \left[x-\frac{1}{2} \pi\left(n+\frac{1}{2}-p\right)\right]$.

Fermer leitet man aus (1), (2) leicht:

$$
J^{-n}(x)=\sqrt{\frac{2}{\pi x}} \Sigma \frac{(n, p)}{(2 x)^{p}} \cos \left[x-\frac{1}{2} \pi\left(-n+\frac{1}{2}-p\right)\right]
$$

ab. Wenn man nun beachtet, dass nach bekannten Reductionsformeln für ein ganzes $p$ :

also

$$
\frac{\Gamma\left(-n+p+\frac{1}{2}\right)}{\Gamma\left(-n-p+\frac{1}{2}\right)}=\frac{\Gamma\left(n+p+\frac{1}{2}\right)}{\Gamma\left(n-p+\frac{1}{2}\right)}
$$

$$
(+n, p)=(-n, p)
$$

so sieht man, dass die Formel für $J^{-n}(x)$ aus der von $J^{+n}(x)$ einfach durch Vertauschung von $n$ mit $-n$ gewonnen wird, und somit Gleichung (3), welche, wie alle übrigen Reihen dieses Paragraphen, zunächst nnter der Voraussetzung eines positiven reellen Theiles von $n$ gewonnen ist, in dem Falle eines negativen reellen Theiles ron $n$ ebenfalls ihre Geltung behält. Da nun (1), (2), (4) lineare Functionen der beiden $J^{+x}(x)$ und $J^{-n}(x)$ sind, so gelten sie anch sämmtlich 
allgemein für alle complexen Werthe von $n$. Dagegen bleibt die andere Voraussetzung, dass $x$ einen positiven reellen Theil habe, noch bestehen; und es ist $\sqrt{x}$ so zu bestimmen, dass sein Werth positiv wird für ein reelles positives $x$.

Um den Charakter obiger Reihen vollständig zu übersehen, so bemerke man, dass sich $J^{n}(x)$ in die beiden Reihen zerlegen lässt:

$$
\begin{aligned}
J^{n}(x) & =\sqrt{\frac{2}{\pi x}} \cos \left[x-\frac{1}{2} \pi\left(n+\frac{1}{2}\right)\right] \Sigma(-1)^{p} \frac{(n ; 2 p)}{(2 x)^{2 p}} \\
& -\sqrt{\frac{2}{\pi x}} \sin \left[x-\frac{1}{2} x\left(n+\frac{1}{2}\right)\right] \Sigma(-1)^{p} \frac{(n, 2 p+1)}{(2 x)^{2 p+1}},
\end{aligned}
$$

und jede von diesen hat den Charakter, dass, wenn man die Reihe an irgend einer Stelle abbricht, ihr Werth von dem complexen Werthe, den sie darstellen soll, nùr um eine Grösse abweicht, deren Modul kleiner ist, als das nächtsfolgende Glied. Jedoch kann diese Eigenschaft nur von dem Gliede an behauptet werden, von dem an $2 p$ oder $2 p+1$ grösser ist, als der reelle Theil von $\left(n-\frac{1}{2}\right)$.

Wenn $n$ und $x$ reelle positive Grössen sind, so haben in jeder Reihe die Glieder von $p \geqq n$ an alternirende Zeichen; denn es wechselt von $p>n$ an das Zeichen in der Reihe der Coefficienten $(n, p)$ regelmässig $a b$, und es haben daher alle $(n, 2 p)$ dasselbe Vorzeichen; ebenso sind alle $(2 p+1)$ desselben Zeichens. Die Reihen sind demnach in diesem Falle semiconvergente Reihen von dem gewöhnlichen Charakter *).

*) Von den Resultaten dieses Paragraphen ist nur Reihe (3) für ganze $n$ und reelle positive $x$ von Jacobi (Schumacher astr. Nachr. 28. p. 94) und Lipschitz (Crellè Journ. to 56. p. 194) auf ühnliche Weise, als oben, und eine Reihe für $Y^{0}(x)$ und $Y^{1}(x)$ von Lommel (Studien üb. d. Bess. F. p. 93) entwickelt worden. Wàs die Ableitung der semiconvergenten Reihe für $J^{n}(x)$ betrifft, die Letzterer gègeben hat, so entspricht sie nicht den Anforderungen, welche die nenere Analyse an eine solche stellt: Nachdem Herr Lommel die endliche Beihe nach absteigenden Potenzen für ein $n=m+\frac{1}{2}$ entwickelt hat, wo $m$ eine ganze Zahl ist, und gezeigt, dass diese Reihe „vermöge ihrer Form allein schon" den Grundeigenschaften der Bessel'schen Functionen genügt, „hält er sich (p. 57) für bereehtigt, die Gleichung auch dann noch als Ausdruck der Bessel'schen Functioneu anzusehen, wenn $m$ gebrochen ist, mit dem Vorbehalte freilich, dass die fragliche umendliche Reihe überhaxpt zulässig sei." Die Bedeutung dieses Vorbehaltes, über den nichts weiter bemerkt wird, kann wohl keine andere sein, als die der Semiconvergenz der Reihe. Wie soll dies aber erwiesen werden, ohne den Rest za entwickeln? da es auf keine Weise mögtich ist, einer Reihe an und für sich anzusehen, ob sie semiconvergent ist, oder nicht; und eine solche Reihe ohne Kenntniss oder wenigstens vorherige Abschätzung des Restes überhaupt keine Bedentang hat. Eine semiconvergente Potenzreike ist nicht, wie eine convergente, ihrer Fo I m nach characteristisch für die entwickelte Function: sie ist nichts weiter, als eine Reihe von Einschliessungen innerhalb endlich von einander, entfernter Grenzen, die man nicht ohne Weiteres für andere Werthe der Veränderlichen in Anspruch nehmen kann, als wofür sie bewiesen ist. 


\section{§. 7.}

\section{Discussion der semiconvergenten Reihen bei Variabilität des $x$.}

Die Reihen des vorigen §. sind zunächst unter der Voraussetzung eines $x$ mit positivem reellen Theile abgeleitet worden, und es entsteht die Frage, ob und inwiefern diese Voranssetzung eine zufällige ist.

$\mathrm{Zu}$ diesem Zwecke denken wir ans in der Reihe aus (1) des vorigen $\S$.

$$
\frac{J^{n}(x)-e^{n \pi i} J^{-n}(x)}{\sin 2 n \pi}=-\frac{i}{\sqrt{2 \pi x}} \frac{i}{\cos n \pi} \Sigma \frac{(n, p)}{(2 x)^{p}} e^{\left[x-\frac{1}{2} \pi\left(n+\frac{1}{2}-y\right)\right]_{i}}
$$

$x$ mit $-x$ vertauscht, wodurch sie in:

$$
+\frac{i}{\sqrt{-2 \pi x}} \frac{e^{\frac{1}{2} \pi i}}{\cos n \pi} \sum \frac{(n, p)}{(2 x)^{p}} e^{-\left[x-\frac{1}{2} \pi\left(n+\frac{1}{2}-p\right)\right] i}
$$

übergeht, welche Reihe nach (2) des §. 6. den Werth:

$$
i \sqrt{\frac{x}{-x}} \frac{e^{n \pi i} J^{n}(x)-J^{-n}(x)}{\sin 2 n \pi}
$$

besitzt. Durch Vertauschung von $x$ mit $-x$ würde aber die linke Seite der Gleichung (1) in

$$
\frac{J^{n}(-x)-e^{n \pi i} J^{-n}(x)}{\sin 2 n \pi}
$$

übergegangen sein, und es fragt sich, ob dies mit (I) überstimmt oder nicht. Man bemerkt nun, dass, wenn die Variabele sich in positiver Rich tung ( $\mathrm{d}$. h. oberhalb am $x=0$ herum) von $+x$ bis zu $-x$ bewegt, $J^{n}(x)$ dadurch den Factor $e^{n \pi i}, J^{-n}(x)$ den Factor $e^{-n \pi i}$ erlangt; wie man aus den Reihenentwicklungen in §. 1. leicht findet. Dann würde also vorstehender Ausdruck

$$
=\frac{e^{n \pi i} J^{n}(x)-J^{-n}(x)}{\sin 2 n \pi}
$$

sein, und da bei derselben Variabilität des $x, \sqrt{-x}=\sqrt{x} e^{\frac{1}{2} \pi i}$, so stimmt dieser Werth mit (I) utberein, d. h. die Gleichung (1) besteht auch noch für ein $x$ mit negativem reellen Theile, wenn man sich $x$ aus dem ursprünglichen Gebiet der Gültigkeit '(dem 4. und 1. Quadranten) auf positivem Wege in den 2. oder 3. Quadranten übergehend denkt.

Die Gleichung (1) besteht jedoch richt mehr, wenn $x$ nach einem vollen Umlaufe um $x=0$ in das ursprüngliche Intervall der Gültigkeit zu seinem anfänglichen Werthe zurückkehrt, 'da unterdessen die Functionen $J^{n}(x)$ und $J^{-2}(x)$ respective die Factoren $e^{2 n \pi i}, e^{-2 n \pi i}$ angenommen haben, die Reihe in (1) aber nur den Factor - 1. Nur in dem Falle, dass $\dot{n}=m+\frac{1}{2}$ und $m$.eine ganze Zahl ist, bleiben beide Seiten auch dann noch einander gleieh; dann aber ist, wie er- 
sichtlich, die Reihe (1) keine semiconvergente Entwickelung, sondern eine endliche Reihe. Wir dürfen von diesem Falle daher hier und im Folgenden ganz absehen.

Die Anwendbarkeit der Formel (1) hört hiernach anf, sobald $x$ aus dem 3. Quadranten in den 4. tritt; und man kann, wie mir scheint, dieses merkwürdige Verhalten am besten so ausdrücken:

Man zerschneide die Ebene von 0 bis - $\infty i$ in der negativ-imaginären Axe, bestimme die vieldeutigen Potenzen $x^{ \pm^{n}}$ in $J^{ \pm n}(x)$ und $\sqrt{x}$ so, dass sie sich für $x=+1$ anf +1 reduciren, so gilt die Gleichung (1) in der ganzen Ebene, so weit sie nicht zerschnitten ist.

Aehnlich, jedoch anders verhält es sicch mit der Gleichung:

$$
e^{n \pi i} \frac{e^{n \pi i} J^{n}(x)-J^{-n}(x)}{\sin 2 n \pi}=\frac{i}{\sqrt{2 \pi x}} \frac{e^{n \pi i}}{\cos n \pi} \sum \frac{(n, p)}{(2 x)^{p}} e^{-\left[x-\frac{1}{2} n\left(n+\frac{1}{2}-p\right)\right] i}
$$

Vertauscht man in der Reihe $x$ mit $-x$, so geht sie über in:

$$
\frac{i}{\sqrt{-2 \pi x}} \frac{e^{\left(2 n+\frac{1}{2}\right) \pi i}}{\cos n \pi} \sum \frac{(n, p)}{(2 x)^{p}} e^{\left[x-\frac{1}{2} \pi\left(n+\frac{1}{2}-p\right)\right] i}
$$

welche nach (1) summirt werden kann und:

$$
=-i \sqrt{\frac{x}{-x}} \cdot e^{n \pi i} \frac{J^{n}(x)-e^{n \pi i} J^{-n}(x)}{\sin 2 n \pi}
$$

ergiebt. Die linke Seite von (2) geht durch diese Vertauschung in:

$$
e^{n \pi i} \frac{e^{n \pi i} J^{n}(-x)-J^{-}(x)}{\sin 2 n \pi}
$$

über, und soll dies mit dem Werthe (II) übereinstimmen, so muss

$$
J^{n}(-x)=e^{-n \pi i} J^{n}(+x), J^{-n}(-x)=e^{+x \pi i} J^{-n^{i}}(+x) \text { und }
$$

$\sqrt{-x}=-i \sqrt{x}$ gesetzt werden, d. h. man muss voraussetzen, dass jetzt $x$, sich in negativem Sinne (unterhalb $x=0$ ) von $+x$ zu $-x$ bewegt hat.

Man erhält daher das Intervall, in dem Gleichung (2) gilt, wenn man sich die Ebene in der positiven imaginären Axe von 0 bis $+\infty i$ zerschnitten denkt. Die Bestimmung der vieldentigen Potenzen erfolgt hierbei so, wie vorhin, dass sie für $x=+1$ der reellen positiven Einheit gleich sind.

Diese Eigenthümlichkeit der semiconvergenten Reihen hăngt mit dem Umstande zusammen, dass sie für die Linien, in welchen wir die Ebenen zerschnitten, weil in ihnen resp. die Gleichungen (1), (2) zu bestehen aufhören, selbst alle Bedeutung verlieren.

Was zuerst (1) anbelangt, so geht diese Reihe für $x=-y i$ in:

$$
\frac{i}{\sqrt{2 \pi y}} \frac{e^{\frac{1}{2} n \pi i}}{\cos n \pi} e^{y} \Sigma(-1)^{p} \frac{(n, p)}{(2 y)^{p}}
$$


über. Wenn nun $y$ positiv reell, so ist diese Reihe nicht mehr semiconvergent, wie man für ein reelles $n$ leicht erkennt; denn für ein solches werden die in der Reihe $(n, p)$ alternirenden Zeichen durch $(-1)^{p}$ aufgehoben und die Reihe enthält von $2 p>n$ an lauter Glieder einerlei Zeichens. Eine Reihe mit lauter positiven Gliedern aber kann die Eigenschaft nicht mehr besitzen, dass sie, an einer beliebigen Stelle abgebrochen, sich von einem gewissen Werthe immer weniger unterschiede, als das nächstfolgende Glied angiebt*).

Was die Reihe (1) für $x=+y i$ betrifft, so geht sie für diesen Werth in

$$
-\frac{1}{\sqrt{2 \pi y}} \frac{e^{\frac{1}{2} n \pi i}}{\cos n \pi} e^{-y} \Sigma \frac{(n, p)}{(2 y)^{p}}
$$

über, also, wenn $n$ reell, in eine Reihe mit abwechselnden Zeichen,

*) Denn angenommen, die aus lauter positiven abnehmenden Gliedern bestehende Reihe

$$
R=u_{0}+u_{1}+u_{2}+\ldots, \text { wo } u_{0}>u_{1}>u_{2}>\ldots
$$

sei semiconvergent, so sei 1$) u_{0}>R$. Dann ist $u_{0}+u_{1}$ jedenfalls $>R$ um eine Grösse, welche $>u_{1}$ ist. Nun soll aber bei einer semiconvergenten Reihe, wenn sie abgebrochen wird, $u_{0}+u_{4}$ sich von $R$ um weniger, als das nächste Glied $u_{2}$ unterscheiden. Dies widerspricht aber vorigem, wonach der Unterschied $>u_{1}$ also um so mehr $>u_{2}$ ist. Wenn 2) $u_{0}<R$ ist, so wäre es doch von $R$ um weniger als $\iota_{1}$ verschieden und daber $u_{0}+u_{1}>R$; dann aber kömnen analoge Betrachturgen, als im ersten Falle angestellt werden.

Eine Reihe mit lanter positiven Gliedern kamn daher, wenigstens an solchen Stellen, wo ihre Glieder abnehmen, nicht semiconvergent sein. Nun kann man aber in der in Text betrachteten Reihe jedenfalls $y$ so gross annehmen, dass bis zu einem beliebigen $p$ die Glieder sämmtlich abnehmen; die Reike könnte demnach bis zu diesem hin nicht als semiconvergent betrachtet werden; und doch würde man gerade die Reihe bis zu dem kleinsten Gliede fortsetzen, um den möglichst hoben Grad von Genauigkeit zu erhalten. Somit kann eine solche Reihe mit lauter positiven Gliedern nar in gewissem Sinne noch als semiconvergent angesehen werden, der mir indess von vorstehender Reihe, die Riemann (Poggendorffs Annal. t. 95. p. 135) als solche in Anspruch nimmt, nicht verständlich ist.

Bei dieser Gelegenheit erlaube ich mir eine höchst einfache, aber, wie mir scheint, noch nicht ansgesprochene Bemerkung zu machen, welche für semiconvergente Reihen mit abwechselnden Vorzeichen - dem am häufigsten vorkommenden Falle - die oft complicirte Abschätzung der Grösse des Restes ganz erspart. Wenn man nämbich nachweisen kam, dass der Rest immer von entgegengesetztem Zeichen, als das letzte Glied, also:

so ist

$$
R=u_{0}-u_{1}+u_{2}-\ldots+(-1)^{n} u_{n}+(-1)^{n+1} r_{n+1},
$$

$(-1)^{n+1} r_{n+1}=(-1)^{n+1} u_{n+1}+(-1)^{a+2} r_{n+2}$ oder $r_{n+1}=u_{n+1}-r_{n+2}$, und somit, wem die $r$ alle positive Grōssen sind, $r_{n+1}>u_{n+1}$.' Eine solehe Reihe ist also inmor semiconvergent.

Wenn die Zeichen nicht rein alternirend sind, wird eine Abschätzang der Grösse des Restes und der Nachweis, dass er kleiner, als das folgende Glied ist, allerdings nöthig sein. 
welche allerdings semiconvergent sein wird. Erinnert man sich jedoch, dass diese Reihe eigentlich als aus der der Glieder von gerader und der yon ungerader Ordnung zusammengesetzt zu betrachten ist, deren jede, für sich genommen, jetzt anch wieder nur Glieder einerlei Zeichens enthält, so scheint jetzt auch vorstehende Reihe unbrauchbar. Indessen lässt sich zeigen, dass man in dem Falle $x=+y i$ die Reihe als Eine zu betrachten hat. Denn in dem Falle, dass $x=y i$ ist, kann an Stelle des Integrales, welches im vorigen §. zur Entwicklung der semiconvergenten Reihe führte, das andere Integral

$$
\int_{1}^{\infty} e^{-y t}\left(t^{2}-1\right)^{2-\frac{1}{2}} d t
$$

genommen werden, welehes durch die Substitution $t=1+r$ in:

$$
e^{-2\left(n-\frac{1}{2}\right) \pi i} 2^{n-\frac{1}{2}} e^{-y} \int_{0}^{\infty} e^{-y r} r^{n-\frac{1}{2}}\left(1+\frac{r}{2}\right)^{n-\frac{1}{2}} d r
$$

übergeht. Jetzt aber $\operatorname{kann}\left(1+\frac{r}{2}\right)^{n-\frac{1}{2}}$ in eine theils convergente, theils semiconvergente Reihe entwickelt werden, welche nicht in zwei zu zerfällen ist; und man erhält so obige Reihe in dem angegebenen Sinne.

In ganz derselben Weise lässt sich denn auch zeigen, dass die Reihe (2) für $x=+y i$, in der Schnittlinie, welche die Gültigkeit der Gleichung (2) begrenzt, aufhört, eine Bedeutung zu haben; wäh. rend sie für $x=-y i$ als Eine Reihe zu fassen, und nicht in zwei zu zerfällen ist.

Nachdem wir so die Gültigkeitsintervalle der Gleichungen (1), (2) kennen gelernt haben, so ist es leicht, zu sehen, dass die aus (1), (2) durch lineare Verbindung entstandenen Formeln (3), (4), (5) des \&. 6 . nur innerhalb des Intervalles bestehen bleiben können, in dem jene beiden Gleiehungen (1), (2) gelten, d. h. also: Es gelten die semiconvergenten Reihen (3), (4) nur für solche $x$, die einen positiven reellen Theil haben, und es ist diese Besehränkung somit nicht eine zufällige, sondern eine wesentliche.

In der That: Vertauscht man in der Reihe:

$$
\sqrt{\frac{2}{\pi x}} \quad \Sigma \frac{(n, p)}{(2 x)^{p}} \cos \left[x-\frac{1}{2} \pi\left(n+\frac{1}{2}-p\right)\right],
$$

welche für $x$ mit positivem reellen Theile $=J^{n}(x)$ ist, $x$ mit $-x$, so dass sie in:

$$
\sqrt{-\frac{2}{\pi x}} \Sigma \frac{(n, p)}{(2 x)^{p}}(-1)^{p} \cos \left[x+\frac{1}{2} \pi\left(n+\frac{1}{2}-p\right)\right]
$$

übergeht, so kann sie nach (4) summirt werden, und es. hat die Reihe (3) für $x$ mit negativem reellen Theile den Werth: 


$$
i \cos n \pi e^{n \pi i} \frac{1}{\pi} Y^{-n}(-x) .
$$

Ebenso findet man, dass die Reihe (4)

$$
\sqrt{\frac{2}{\pi x}} \Sigma \frac{(n, p)}{(2 x)^{2}} \sin \left[x-\frac{1}{2} \pi\left(n+\frac{1}{2}-p\right)\right] \text {, }
$$

die für $x$ mit positivem reellen Theile den Werth:

$$
\cos n \pi e^{-n \pi i} \frac{1}{\pi} Y^{n}(x),
$$

hat, für solche mit negativem reellen' Theile den Werth:

annimmt.

$$
i J^{-n}(-x)
$$

Um demnach auch für solche $x$, welche einen negativen reellen Theil haben, eine semiconvergente Entwickelung zu erhalten, hat man $J^{n}(x)$ erst auf $J^{n}(-x)$, in dem also die Variabele $(-x)$ einen positiven reellen Theil hat, mittels der Gleichung:

$$
J^{n}(x)=e^{n \pi i} J^{n}(-x)
$$

zu reduciren. Für rein imaginäre $y$ aber ist eine Entwickelung dieser Art nicht statthaft.

Während also:

$$
\sqrt{\frac{2}{\pi x}} \cos \left[x-\frac{1}{2} \pi\left(n+\frac{1}{2}\right)\right]
$$

der asymptotische Werth von $J^{n}(x)$ für $x$ mit reellem positiven Theile ist, wo $\sqrt{x}$ mit reellem positiven Theile zu nehmen ist, so nähert sich $J^{n}(x)$ für solche $x$, die einen negativen reellen Theil haben, dem Ausdruck:

$$
e^{n \pi i} \sqrt{\frac{2}{-\pi x}} \cos \left[x+\frac{1}{2} \pi\left(n+\frac{1}{2}\right)\right],
$$

wenn der Modul unendlich wächst; und es ist $\sqrt{-x}$ für reelle negative $x$ positiv zu nehmen.

Wenn $x=\varepsilon+y i$ gesetzt wird, wo $\varepsilon$ eine unendlich abnehmende, $y$ eine unendlich zunehmende reelle positive Grösse bezeichnet, so ist nach der ersten Formel der asymptotische Werth von $J^{n}\left(\varepsilon+y^{i}\right)$

wo

$$
\sqrt{\frac{1}{2 \bar{\pi}(\bar{\varepsilon}+\overline{y i})}} e^{\frac{1}{2\left(x+\frac{1}{2}\right) \pi i}} e^{y}
$$

$$
\sqrt{\varepsilon+y^{i}}=\sqrt{\sqrt{\varepsilon^{2}+y^{2}}} e^{\frac{1}{z} i \arctan \frac{y}{\varepsilon}},
$$

und in $\frac{1}{2}$ are tan $\frac{y}{\varepsilon}$ der Bogen im 1. Quadranten zu nehmen ist, damit der reelle Theil von $\sqrt{\varepsilon+y i}$ positiv sei. Nimmt nun $\varepsilon$ unendlich $\mathbf{a b}$, so wird are tan $\frac{y}{\varepsilon}=\frac{\pi}{2}$ und $\sqrt{\sqrt{\varepsilon^{2}+y^{2}}}=\sqrt{y}$, so dass; 


$$
\sqrt{\frac{1}{2 \pi y}} e^{\frac{1}{2} n \pi i} e^{y}
$$

der asymptotische Werth von $J^{n}(\varepsilon+y i)$ ist.

Um den von $J^{n}(-\varepsilon+y i)$ zu bestimmen, nehmen wir

$$
J^{n}(-\varepsilon+y i)=e^{n \pi i} J^{n}(\varepsilon-y i)
$$

und finden so:

$$
e^{n \pi i} \sqrt{\frac{1}{2 \pi(\varepsilon-y i)}} e^{-\frac{1}{2}\left(n+\frac{1}{2}\right) \pi i} e^{y}
$$

als asymptotischen Werth; jetzt ist:

$$
\sqrt{\varepsilon-y i}=\sqrt{\sqrt{\varepsilon^{2}+y^{2}}} e^{-\frac{1}{2} i \arctan \frac{y}{\varepsilon}}
$$

und bei unendlich abnehmenden $\varepsilon, \arctan \frac{y}{\varepsilon}=\frac{\pi}{2}$ zu setzen, so dass man

$$
\sqrt{\frac{1}{2 \pi y}} e^{\frac{1}{2} m \pi i} e^{y}
$$

als asymptotischen Werth von $J^{+n}(-\varepsilon+y i)$ findet. Da dieser mit dem von $J^{n}(\varepsilon+y i)$ übereinstimmt, so können wir ihn sofort für den asymptotischen Werth von $J^{n}(y i)$ mit unendlich wachsendem $y$ überhaupt annehmen.

Bei richtiger Bestimmung der Zeichen ist daun:

$$
\sqrt{\frac{1}{2 \pi y}} e^{-\frac{1}{2} n \pi i} e^{y}
$$

der asymptotische Werth von $J^{n}(-y i)$.

Erlangen, 15. December 1868. 